\title{
Graft IL-33 regulates infiltrating macrophages to protect against chronic rejection
}

\author{
Tengfang Li, ${ }^{1,2,3}$ Zhongqiang Zhang, ${ }^{1,2,4}$ Joe G. Bartolacci, ${ }^{1,5}$ Gaelen K. Dwyer, ${ }^{1,2,6}$ Quan Liu, ${ }^{1,2,7}$ Lisa R. Mathews, ${ }^{1,2}$ \\ Murugesan Velayutham, ${ }^{1,2,8,9}$ Anna S. Roessing, ${ }^{1,2,6}$ Yoojin C. Lee, ${ }^{5,10}$ Helong Dai, ${ }^{1,2,3}$ Sruti Shiva, ${ }^{8,9}$ Martin H. Oberbarnscheidt, ${ }^{1,2,6}$ \\ Jenna L. Dziki, ${ }^{1,5}$ Steven J. Mullet, ${ }^{10,11,12}$ Stacy G. Wendell, ${ }^{9,10,11,12}$ James D. Wilkinson, ${ }^{13}$ Steven A. Webber, ${ }^{13}$ \\ Michelle Wood-Trageser, ${ }^{2,14}$ Simon C. Watkins, ${ }^{15}$ Anthony J. Demetris, ${ }^{2,5,14}$ George S. Hussey, ${ }^{1,5}$ Stephen F. Badylak, ${ }^{1,5,10}$ \\ and Hēth R. Turnquist ${ }^{1,2,5,6}$
}

'Department of Surgery and ${ }^{2}$ Thomas E. Starzl Transplantation Institute, University of Pittsburgh School of Medicine, Pittsburgh, Pennsylvania, USA. ${ }^{3}$ Department of Kidney Transplantation and ${ }^{4}$ Department of Organ Transplantation and General Surgery, Second Xiangya Hospital of Central South University, Changsha, China. ${ }^{5}$ McCowan Institute for Regenerative Medicine and ${ }^{6}$ Department of Immunology, University of Pittsburgh School of Medicine, Pittsburgh, Pennsylvania, USA. 'Southern University of Science and Technology, Shenzhen, China. ${ }^{8}$ Pittsburgh Heart, Lung, and Blood, Vascular Medicine Institute and ${ }^{9}$ Department of Pharmacology and Chemical Biology, University of Pittsburgh School of Medicine, Pittsburgh, Pennsylvania, USA. ${ }^{10}$ Department of Bioengineering, University of Pittsburgh, Pittsburgh, Pennsylvania, USA. "Health Sciences Metabolomics and Lipidomics Core and ${ }^{12 C}$ Clinical Translational Science Institute, University of Pittsburgh School of Medicine, Pittsburgh, Pennsylvania, USA. ${ }^{3}$ Department of Pediatrics, Vanderbilt School of Medicine, Nashville, Tennessee, USA. ${ }^{14}$ Department of Pathology and ${ }^{15}$ Department of Cell Biology, University of Pittsburgh School of Medicine, Pittsburgh, Pennsylvania, USA.

\begin{abstract}
Alarmins, sequestered self-molecules containing damage-associated molecular patterns, are released during tissue injury to drive innate immune cell proinflammatory responses. Whether endogenous negative regulators controlling early immune responses are also released at the site of injury is poorly understood. Herein, we establish that the stromal cell-derived alarmin interleukin 33 (IL-33) is a local factor that directly restricts the proinflammatory capacity of graft-infiltrating macrophages early after transplantation. By assessing heart transplant recipient samples and using a mouse heart transplant model, we establish that IL-33 is upregulated in allografts to limit chronic rejection. Mouse cardiac transplants lacking IL-33 displayed dramatically accelerated vascular occlusion and subsequent fibrosis, which was not due to altered systemic immune responses. Instead, a lack of graft IL-33 caused local augmentation of proinflammatory iNOS+ macrophages that accelerated graft loss. IL-33 facilitated a metabolic program in macrophages associated with reparative and regulatory functions, and local delivery of IL-33 prevented the chronic rejection of IL-33-deficient cardiac transplants. Therefore, IL-33 represents what we believe is a novel regulatory alarmin in transplantation that limits chronic rejection by restraining the local activation of proinflammatory macrophages. The local delivery of IL-33 in extracellular matrix-based materials may be a promising biologic for chronic rejection prophylaxis.
\end{abstract}

\section{Introduction}

Acute heart transplant rejection can typically be averted by immunosuppressant therapy, which controls recipient $\mathrm{CD}^{+}$and $\mathrm{CD} 8^{+}$ $\mathrm{T}$ cell responses to polymorphic amino acids in donor MHC molecules or donor-derived peptides presented on recipient MHC $(1$, 2). However, immunosuppressants are ineffective against development of chronic heart transplant rejection, a progressive vascular and fibrotic remodeling that leads to progressive myocardial dysfunction and loss of the majority of grafts within approximately 11 years after transplant (3-5). Although the immunopathology of chronic rejection is not fully understood, experimental stud-

Authorship note: $T L$ and $Z Z$ share the first author position. Conflict of interest: SFB, JLD, and CSH are shareholders in ECM-Therapeutics, Inc. JLD, ZZ, GSH, SFB, HRT, and QL are listed inventors on patent application PCT/ US2019/030547 ["Matrix bound vesicles (MBV) containing IL-33 and their use"] Copyright: (5) 2020, American Society for Clinical Investigation.

Submitted: August 29, 2019; Accepted: July 7, 2020; Published: September 14, 2020 Reference information: J Clin Invest. 2020;130(10):5397-5412. https://doi.org/10.1172/JCl133008. ies have clearly established that chronic rejection of heart transplants, manifesting as vessel occlusion and myocardial fibrosis, is an immunologically driven process that does not occur in the absence of adaptive immune cells, particularly T cells (6). Clinical studies have indicated the importance of alloimmune response to human leukocyte antigen (HLA), as patients transplanted with cardiac grafts with HLA mismatching are reported to have higher incidence of chronic allograft vasculopathy (CAV), with HLA-DR mismatching being the most important HLA subtype $(7,8)$. The number of acute rejection episodes and total rejection score are positively related to development of CAV in both human and animal models $(9,10)$. Anti-HLA and anti-endothelial antibodies are also implicated in development of CAV $(11,12)$.

While chronic rejection is $\mathrm{T}$ and $\mathrm{B}$ cell mediated, innate immune cells, including natural killer (NK) cells, inflammatory macrophages, monocytes, and monocyte-derived dendritic cells (monoDCs) initiate and sustain the adaptive responses leading to chronic rejection. Chronic heart rejection does not occur in $\mathrm{RAG}^{-/}$ mice lacking $\mathrm{T}$ and $\mathrm{B}$ cells, yet NK cells predisposed allografts to adoptively transferred, $\mathrm{T}$ cell-mediated chronic rejection (13). 
Likewise, kidney and heart allografts are rapidly infiltrated with recipient monocytes that differentiate into inflammatory macrophages and monoDCs, which act as critical local stimuli to alloreactive T cells (14). Early macrophage depletion (15) and targeting macrophage mammalian target of rapamycin (mTOR) signaling $(16,17)$ suppress cardiac allograft vasculopathy in mice. Given that $\mathrm{T}$ cell-centric immunosuppressants fail to prevent chronic rejection, the development of new therapeutics able to limit the early response of innate immune cells after transplant surgery may be an effective tool to improve long-term outcomes after heart transplant, which have not changed significantly in the last 20 years (5).

It is well appreciated in transplantation that ischemia/reperfusion injury (IRI) arising after abrupt disruption and reestablishment of the blood supply to the solid organ results in myeloid cell infiltration, differentiation, and proinflammatory activation in the graft (18). Like IRI, surgical procedures, recipient conditioning, or alloimmune responses also cause tissue damage or nonapoptotic cell death that releases sequestered self-derived molecules, or alarmins, to alert the immune system (18). There have been numerous proinflammatory alarmins identified, such as ATP, mitochondrial contents, and DNA. However, the best-characterized alarmin is high-mobility group box 1 (HMGB1) (18). HMGB1 stimulates myeloid cells through TLR4 and RAGE to promote the expression of costimulatory molecules and proinflammatory cytokines. These mechanisms have been shown to support acute and chronic rejection in experimental models $(16,18-20)$ and have been implicated clinically in acute liver graft rejection (21) and chronic heart transplant rejection (22). HMGB1 is upregulated by IRI in cadaveric kidney transplants but absent in living donor grafts, and recipients having a TLR4 mutation decreasing the affinity for HMGB1 have higher rates of immediate graft function (23). This finding is also consistent with the observation that despite HLA mismatch, recipients of live, unrelated donor kidneys have significantly better long-term outcomes relative to those receiving HLA-matched cadaveric kidney grafts subjected to longer periods of ischemia (24). Recently, it was shown that HMGB1 upregulated in mouse cardiac tissue following IRI induces a metabolic profile and epigenetic modification in myeloid cells that supports proinflammatory cytokine secretion (16). Inhibition of HMGB1-augmented glycolysis using mTOR inhibition directed at myeloid cells using nanovesicles prolonged allograft survival (16). Thus, ischemic injury releasing proinflammatory alarmins sets the stage for long-term outcomes through effects on myeloid cells.

However, there is limited evidence that not all alarmins are proinflammatory and some may actually have beneficial functions after transplantation by stimulating tissue repair or supporting immune regulation (25). Interleukin 33 (IL-33) is an IL-1 family member and alarmin that is typically sequestered in the nucleus (26) or extracellular matrix (ECM) (27) of stromal cells. When released after tissue damage, IL-33 signals to immune cells via the IL-33 receptor IL-1R-like 1 (IL1RL1), more commonly referred to as serum stimulation-2 (ST2) (26). Our work, and that of others, has shown that administration of recombinant IL-33 (rIL-33) after heart transplant prolongs allograft survival across MHC barriers in rodent heart transplant models $(28,29)$. In further studies, we established that a dominant mechanism of action supporting the therapeutic benefit of rIL-33 was immune regulation through sys- temic expansion of ST2 ${ }^{+}$Tregs $(29,30)$. IL-33 expressed by fibrogenic/adipogenic progenitors in skeletal muscle has also been shown to regulate skeletal muscle Treg homeostasis and support muscle regeneration (31). Related studies have suggested a direct, cardioprotective role for rIL-33 against hypertrophy resulting from cardiac overload (32) and fibrosis after myocardial infarction (33). However, delivery of rIL-33 also aggravates autoimmune eosinophilic pericarditis during coxsackievirus B3 infection (34), suggesting that IL-33 can contribute to cardiac inflammation. IL-33 expression has been reported in cardiac fibroblasts (32) and the vasculature (35), yet how the expression of this alarmin is modulated in cardiac allografts or impacts outcomes was unknown.

Using IL-33-deficient heart grafts in a mouse chronic rejection model we have established that IL-33 stands out among identified alarmins and limits differentiation of proinflammatory macrophages to prevent chronic rejection. Specifically, transplants lacking IL-33 displayed dramatically accelerated chronic rejectionassociated vasculopathy and subsequent fibrosis orchestrated by graft-infiltrating recipient proinflammatory macrophages. IL-33expressing heart grafts in recipients with ST2-deficient macrophages also displayed increased graft infiltration by proinflammatory macrophages and accelerated graft loss. Mechanistic studies demonstrated that IL-33 promoted a reparative macrophage phenotype through a metabolic reprograming involving augmented oxidative phosphorylation (OXPHOS) and fatty acid (FA) uptake. We also revealed that IL-33 prevents proinflammatory stimuli-induced disruption of the tricarboxylic acid (TCA) cycle that shifts macrophage metabolism to anaerobic glycolysis and generates proinflammatory metabolites $(36,37)$. Restoration of IL-33 to IL-33-deficient heart transplants using vesicles in ECM-derived hydrogel immediately after transplantation profoundly reduced the frequency of proinflammatory myeloid cells in the graft and prevented graft loss to chronic rejection. Thus, the local delivery of IL-33 in ECM-based materials after transplantation may be a practical and promising biologic for chronic rejection prophylaxis.

\section{Results}

IL-33 is augmented in the allograft and circulation during heart transplant rejection. To define how endogenous IL-33 shapes cardiac transplantation outcomes, we crossed C57BL/6 (B6) IL-33knockout mice (38) onto the $\mathrm{B} 6 \mathrm{H} 2-\mathrm{Ab}^{\mathrm{Bm} 12}$ (Bm12) background. Bm12 mice are a variant strain of B6 mice in which a spontaneous mutation in the third hypervariable region of the $\beta 1$ domain results in alteration of 3 amino acids to generate a distinct I-A molecule, I- $\mathrm{A}^{\mathrm{bm} 12}$ (39). When transplanted into B6 mice, this MHCII molecule is recognized by alloreactive immune cells, particularly $\mathrm{CD} 4^{+}$ $\mathrm{T}$ cells. These responses do not typically result in early acute rejection of Bm12 allografts, but instead result in chronic rejection-associated fibrosis and vasculopathy in contracting grafts at late time points (>30-100 days after transplant) $(40,41)$. In using this model we were able to assess the impact of IL-33 on the alloimmune responses shaping chronic rejection in the absence of immunosuppression.

When $\mathrm{Il33} 3^{-/-} \mathrm{Bm} 12$ and $\mathrm{Il33} 3^{+/+} \mathrm{Bm} 12$ hearts were transplanted into $\mathrm{Il33} 3^{+/+} \mathrm{B} 6$ recipients and assessed using quantitative immunofluorescence on postoperative day (POD) 3 and POD 94, we found a rapid increase in $\mathrm{IL}-33^{+}$cells that was sustained above baseline 
A

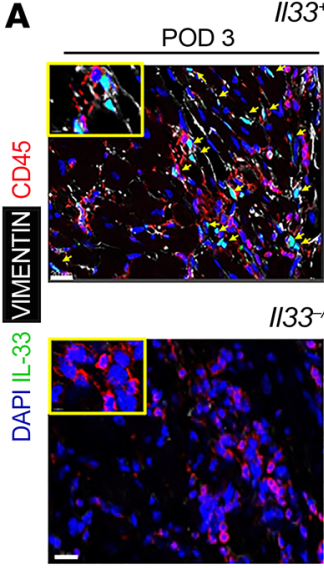

B

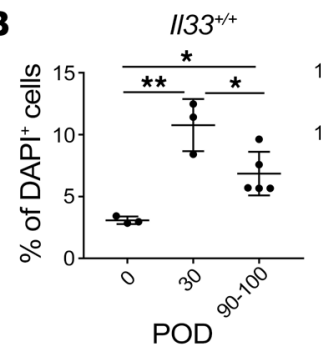

$1133^{++4} \mathrm{Bm} 12$

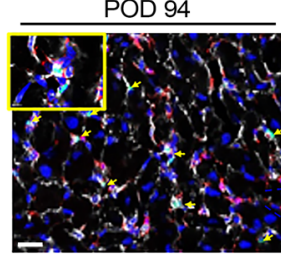

$133^{-}-\mathrm{Bm} 12$

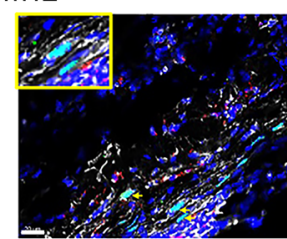

C
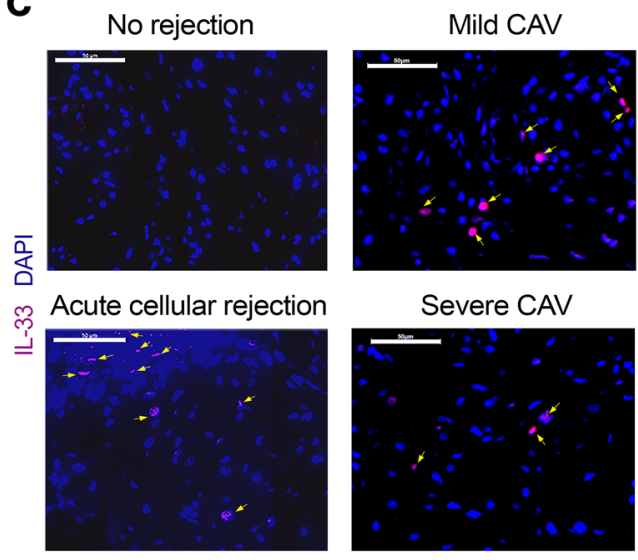

Severe CAV

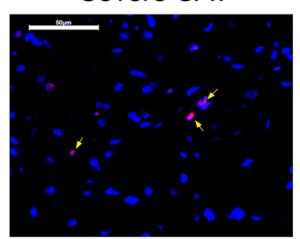

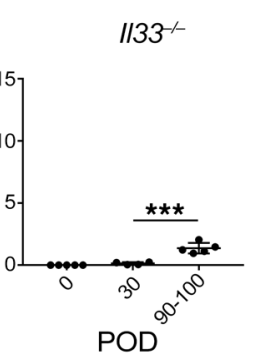
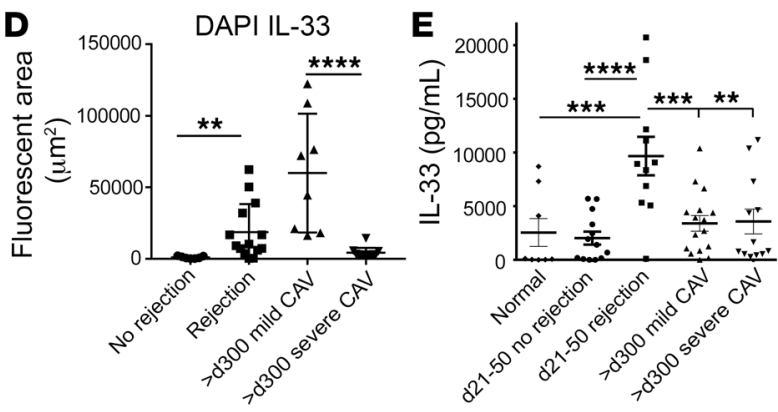

Figure 1. Augmented IL-33 is observed in allografts and the circulation during heart transplant rejection. (A and B) IL-33-expressing (//33+/+) or IL-33deficient (//33-/-) Bm12 grafts were transplanted into WT C57BL/6 (B6) mice ( $n=3-5 /$ group). (A) On postoperative day (POD) 3 or POD 90-100, grafts were evaluated by immunofluorescent staining for CD45 (red), vimentin (white), and IL-33 (green). DAPI (blue) was used to stain nuclei. One representative image for each group is shown. Yellow arrows signify IL-33+ cells. Scale bars: $20 \mu \mathrm{m}$. (B) Percentage IL-33+ cells in relation to the total number of DAPI+ cells. Graphs depict individual values and group mean \pm SD. (C and D) Increased graft IL-33 during acute clinical rejection and chronic rejection-associated coronary artery vasculopathy (CAV) was observed in analysis of endomyocardial biopsies (EMBs) immunostained for IL-33 (magenta) and stained with DAPI (blue). (C) Representative image captures from EMBs at time points diagnosed as rejection-free (top left panel), suffering acute cellular rejection (ACR) (bottom left panel), mild CAV (top right panel), or severe CAV (bottom right panel). Yellow arrows signify IL-33+ cells. Scale bars: $50 \mu \mathrm{m}$. (D) Cohort mean for IL-33+DAPI fluorescent area from EMB samples at times of "No rejection"; ACR or antibody-mediated "Rejection," "Mild" or "Severe CAV" calculated for all readable EMB areas for each subject. d, day. (E) Serum assessed for IL-33 by ELISA grouped by clinical status and time point of collection relative to transplantation. Graphs depict sample values and group means \pm SEM. ${ }^{*} P<0.05 ;{ }^{* *} P<0.01 ;{ }^{* *} P<0.005 ;{ }^{* * *} P<0.0001$ by 1 -way analysis of variance (ANOVA).

levels throughout the life of the transplant (Figure 1, A and B, and Supplemental Figure 1; supplemental material available online with this article; https://doi.org/10.1172/JCI133008DS1). Consistent with other reports $(31,42)$, graft IL-33+ cells were predominantly $\mathrm{CD}^{4} 5^{-}$vimentin $^{+}$stromal cells. Interestingly, while $\mathrm{Il33^{-/- }}$ Bm12 IL-33-knockout grafts lacked IL-33+ cells at POD 3, there was a small fraction of $\mathrm{CD} 45^{-} \mathrm{IL}-33^{+}$cells at late time points. These data suggest that recipient-derived cells, most likely fibrocytes (43, 44), can also contribute IL-33 to the transplant microenvironment. Although IL-33+ leukocytes have been described (26), there was a lack of evidence for significant levels of CD $45^{+} \mathrm{IL}-33^{+}$leukocytes in the transplanted grafts (Figure 1A).

We next assessed if a similar modulation of IL-33 was observed clinically by quantifying the level of IL-33 expressed in endomyocardial biopsies (EMBs) and circulating in the serum of pediatric heart transplant recipients. Comparing the expression of IL-33 in EMBs in the first year after transplant at times of pathologistdiagnosed acute cellular rejection (ACR; International Society for Heart and Lung Transplantation grade $\geq 2 \mathrm{R}$ ) or antibody-mediated rejection ( $A M R \geq 2$ ) episodes to those that were deemed rejection free revealed that periods of diagnosed clinical rejection were asso- ciated with increased IL-33 ${ }^{+}$cells in the graft (Figure 1, C and D). Thus, these data paralleled the above findings in our rodent heart transplant model. IL-33 levels were also compared between EMBs from recipients suffering severe or mild chronic rejection-associated CAV at least 300 days after transplant. These limited data suggested that those with more severe CAV had decreased levels of IL-33 (Figure 1, C and D). Assessment of recipient serum revealed that there was a large increase in circulating IL-33 in recipients suffering a diagnosed early rejection event compared with recipients deemed rejection free during a similar time period, which were similar to normal levels (Figure 1E). Later time points also showed a return to normal IL-33 levels, including serum from recipients with severe CAV (Figure 1E). In total, these data showed that endogenous IL-33 is modulated during clinical and experimental heart transplant rejection and free IL-33 is available during early rejection to shape local and systemic immune responses. In addition, that IL-33 remained increased in the grafts of recipients exhibiting less CAV is suggestive of a beneficial role for sustained local IL-33.

Heart transplants lacking IL-33 undergo augmented chronic rejection and increased infiltration by $T$ cells. To delineate the effect of graft or recipient IL-33 on heart transplant outcomes in this model 
A

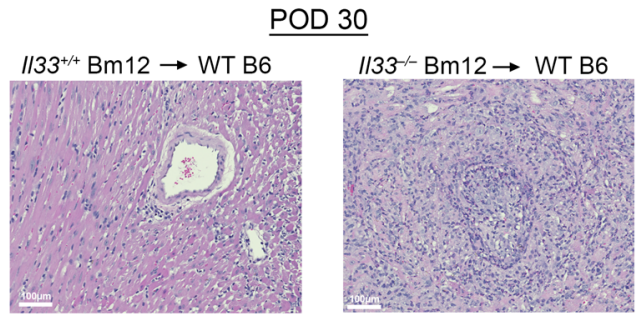

B

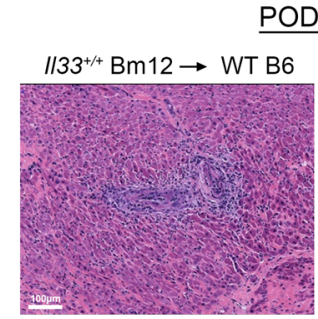

$1133^{--} \mathrm{Bm} 12 \rightarrow \mathrm{WT} \mathrm{B} 6$
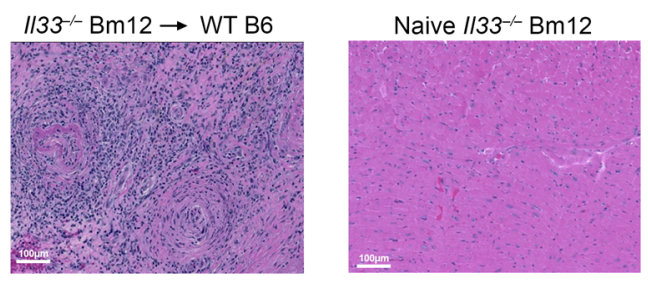

E

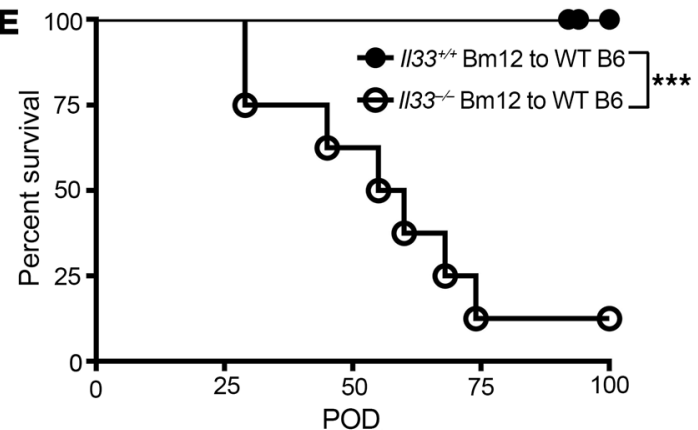

Figure 2. The absence of graft IL-33 results in increased chronic rejection-associated vasculopathy, fibrosis, and T cell infiltration. IL-33-expressing $\left(1 / 33^{+/+}\right)$or IL-33-deficient Bm12 $\left(1 / 33^{-/}\right)$grafts were transplanted into $/ 133^{+/+}$or $1 / 33^{-/-} \mathrm{B} 6$ recipients ( $n=6-7 /$ group). (A-D) Grafts harvested on POD 30 and POD 90-100 were evaluated after H\&E staining for percentage vascular occlusion and fibrotic area quantified using NearCYTE. Graphs depict individual values and group mean $\pm \mathrm{SD}$. Naive $1 / 33^{-1-} \mathrm{Bm} 12$ hearts were stained as controls. Scale bars: $100 \mu \mathrm{m}$. (E) Survival of $1133^{-1-}(n=10)$ versus $1 / 33^{+/+} \mathrm{Bm} 12$ grafts $(n=7)$ in WT B6 recipients. (F-I) Numbers of graft-infiltrating $\mathrm{CD}^{+}$, $\mathrm{Foxp}^{+}$, and $\mathrm{CD}^{2} \mathrm{~b}^{+}$cells identified using immunohistochemistry and quantified with NearCYTE. Graphs depict individual values and group means $\pm \mathrm{SD}$. ${ }^{*} P<0.05$; ${ }^{* *} P$ $<0.005$; ${ }^{* * *} P<0.0001$ by 1 -way ANOVA (C, D, and $\mathbf{F}-\mathbf{I}$ ) or Kaplan-Meier analysis (E).

of chronic rejection, we completed heterotopic transplantation of $\mathrm{Il} 33^{+/+}$or $\mathrm{Il} 33^{-/-} \mathrm{Bm} 12$ hearts in $\mathrm{Il} 33^{+/+} \mathrm{B} 6$ mice and assessed groups of grafts at POD 30 and POD 90-100. A group of $I l 33^{-/} \mathrm{Bm} 12$ hearts transplanted into $I l 33^{-1-} \mathrm{B} 6$ mice was also analyzed at POD

C
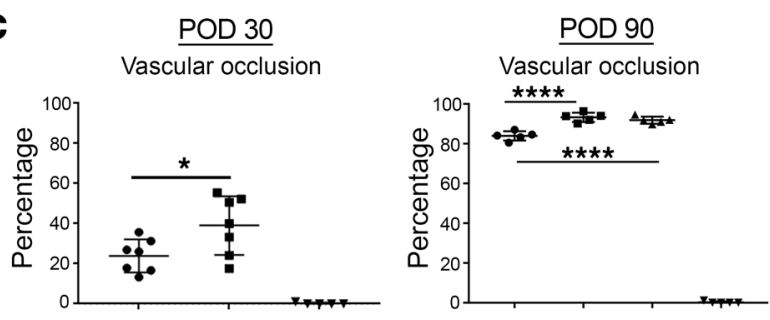

D
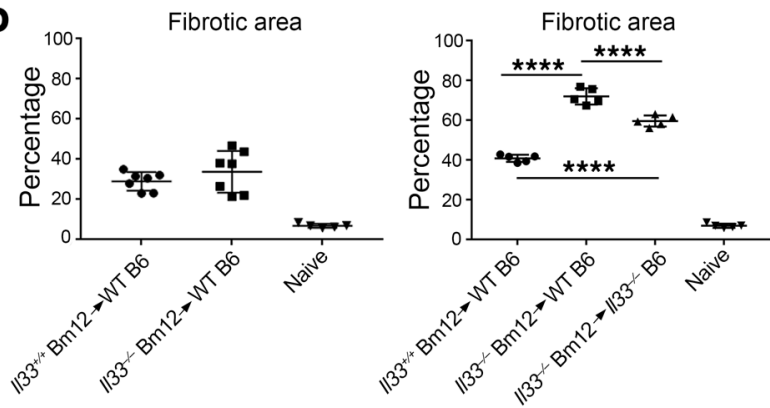

POD 30
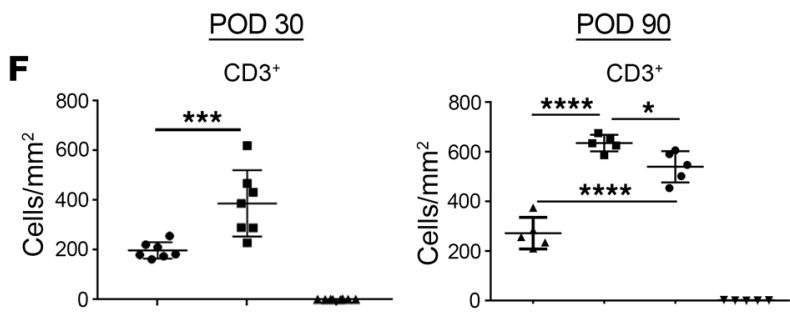

G

Foxp $3^{+}$
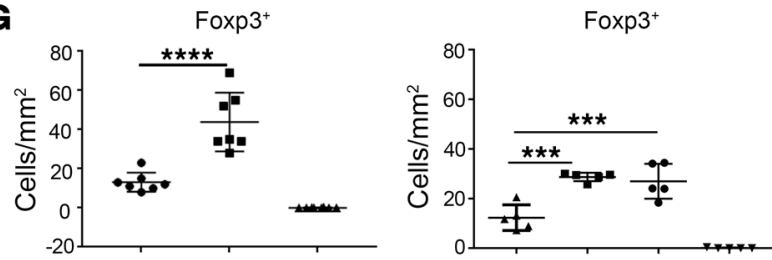

H
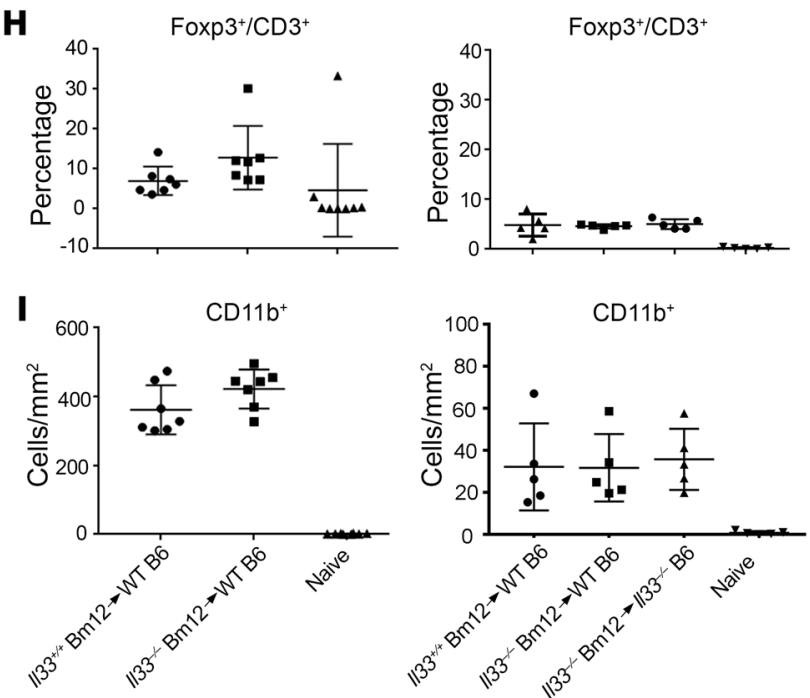

90. The absence of graft IL-33 resulted in increased graft pathology and immune infiltration at both time points (Figure 2, A-I). Using computer-aided image analysis of whole slide images of H\&E(Figure 2B) and trichrome-stained samples (Supplemental Figure 
2, A and B) we established increased early vascular occlusion in $\mathrm{Il33} 3^{-/-} \mathrm{Bm} 12$ hearts transplanted into either $\mathrm{Il33} 3^{+/+} \mathrm{B} 6$ or $\mathrm{Il33} 3^{-/-}$ B6 recipients (Figure 2, A and C). Both vasculopathy and fibrotic disease were increased at the POD 90-100 time point in Il33/$\mathrm{Bm} 12$ hearts transplanted into either $\mathrm{Il33^{+/+ }}$ or $\mathrm{Il33^{-/- }} \mathrm{B} 6$ recipients (Figure 2, B-D). Graft survival reflected the above observations of increased pathology and immune infiltrate, as the absence of graft IL-33 resulted in the accelerated loss of Bm12 heart graft function (Figure 2E). Although $\mathrm{Il33}{ }^{+/+} \mathrm{Bm} 12$ grafts exhibited longterm functioning as expected $(40,41), I l 33^{-/-} \mathrm{Bm} 12$ grafts instead showed late rejection (median survival time $=57.5$ days).

We also used immunohistochemistry to detect CD3, CD11b, and Foxp 3 to characterize the immune infiltrate present at these time points. These assessments revealed that the absence of local IL-33 resulted in increased $\mathrm{CD}^{+}$and $\mathrm{Foxp}^{+}$cell infiltrate but did not alter the frequency of $\mathrm{CD} 11 \mathrm{~b}^{+}$cells (Figure 2, F-I, and Supplemental Figure 2, C-E). Although previous studies have suggested that IL-33 is important for Treg accumulation in injured skeletal muscle (31), graft IL-33 did not appear to modulate local Treg frequency, as their numbers were increased in $\mathrm{Il33^{-/- }} \mathrm{Bm} 12$ relative to $\mathrm{I} l 33^{+/+} \mathrm{Bm} 12$ and appeared to be part of an overall increase in $\mathrm{CD}^{+}$cells in the absence of IL-33 (Figure 2, F-H, and Supplemental Figure 2, C-E).

Although we detected the emergence of limited recipient IL-33+ cells in the graft by POD 90-100, these cells appeared to have minimal functional impact, as there was no significant difference seen in the level of immune infiltration or vascular occlusion between $\mathrm{Il33^{-/- }} \mathrm{Bm} 12$ grafts in $\mathrm{Il33^{+/+ }}$ or $\mathrm{Il33} 3^{-/-} \mathrm{B} 6$ recipients (Figure $2, B-G)$. There was a slight decrease in fibrotic area observed when both the graft and recipient lacked IL-33, suggesting a potential profibrotic role for recipient IL-33 (Figure 2D). In total, these data provided evidence that graft-derived IL-33 acts as a regulatory alarmin that limits chronic rejection.

Graft IL-33 does not alter systemic immune responses after heart transplantation. We next assessed if the lack of IL-33 in the graft affected splenic lymphoid cells at POD 3, POD 30, and POD 90-100 by flow cytometry. Analysis at the early time point did not uncover any global differences in the frequencies of $\mathrm{CD}^{+} \mathrm{T}$ cells, $\mathrm{B}$ cells, or $\mathrm{CD}_{11 \mathrm{~b}^{+}}$myeloid cells between spleens of WT B6 recipients of $\mathrm{Il33^{-/ }}$ or $\mathrm{Il33^{+/+ }} \mathrm{Bm} 12$ heart grafts at POD 3 (Supplemental Figure $3, \mathrm{~A}$ and $\mathrm{B})$. Nor were there differences in the frequency of naive $\left(\mathrm{CD} 44^{-} \mathrm{CD}^{6} 9^{-} \mathrm{CD} 62 \mathrm{~L}^{+} \mathrm{KLRG} 1^{-}\right) \mathrm{CD} 4^{+} \mathrm{T}$ cells or $\mathrm{CD} 4^{+} \mathrm{T}$ effectors $\left(\mathrm{CD} 44^{+} \mathrm{CD} 69^{+} \mathrm{CCR} 7^{-}{ }^{-} \mathrm{KLG} 1^{-}\right)$between WT $\mathrm{B} 6$ recipients of $\mathrm{Il33}{ }^{-/}$or $\mathrm{Il33^{+/+ }} \mathrm{Bm} 12$ heart grafts at POD 30 (data not shown). Flow analyses of WT B6 recipient spleen cells at POD 90-100 revealed that the anticipated (41) increase in $\mathrm{CD}^{+} \mathrm{T}$ effector memory $\left(\mathrm{CD} 4{ }^{+} \mathrm{CCR} 7{ }^{\mathrm{lo}} \mathrm{CD} 44^{+}\right)$cells (Supplemental Figure 4, A and B) was similar between WT B6 recipients of $\mathrm{Il33^{-/- }}$ or $\mathrm{Il33^{+/+ }} \mathrm{Bm} 12$ heart grafts. Thus, the increased graft pathology observed in $\mathrm{Il33}^{-1}$ Bm12 transplants at either POD 30 or POD 90-100 did not appear to be associated with significant differences in systemic recipient $\mathrm{CD}^{+}{ }^{+} \mathrm{T}$ cell response to $\mathrm{H} 2-\mathrm{Ab} 1^{\mathrm{Bm} 12}$.

Although chronic rejection-associated fibrosis and vasculopathy of Bm12 allografts takes place in the presence of $\mathrm{CD}^{+}{ }^{+} \mathrm{Foxp}^{+}$ Tregs, an increase in the presence and the suppressive function of Tregs is important for the long-term survival of Bm12 heart transplants (40). However, in spite of ample evidence that delivery of
rIL-33 produces systemic increases in Tregs that protect cardiac allografts $(29,45)$, the absence of IL-33 in the allograft did not impact the early presence of $\mathrm{ST}_{2}{ }^{+}$or ST2 $2^{-}$Tregs in the spleen at POD 3 (Supplemental Figure 3, A and B). We also observed the expected systemic increases in Foxp $3^{+}$Tregs, including the ST2 ${ }^{+}$ subset, at POD 30 and POD 90-100 of both $\mathrm{Il}_{33^{-/-}}$and $\mathrm{Il33^{+/+ }}$ Bm12 grafts (Supplemental Figure 4, C-F).

Local IL-33+ regulates proinflammatory myeloid cells in the graft cells early after heart transplantation. The heart transplant data to this point indicate that $\mathrm{IL}^{-} 33$ is limiting intragraft $\mathrm{CD}^{+}$cells and early vasculopathy, but not shaping systemic $\mathrm{CD} 4^{+} \mathrm{T}$ cell responses, to temper chronic allograft rejection. The importance of trafficking donor and recipient myeloid cells to the secondary lymphoid tissues where they prime alloreactive $\mathrm{T}$ cells, which then leave to attack the graft, is appreciated $(46,47)$. In addition to acting as dominant drivers of local proinflammatory responses and tissue injury after cardiac IRI $(48,49)$, data have emerged showing that recruited myeloid cells are also critical for sustaining early $\mathrm{T}$ cell responses in the allografts $(14,47)$. Guided by these data, we examined if graft-derived IL-33 was influencing graft or systemic myeloid cell responses after cardiac transplantation. Although graft (Figure 2I, Supplemental Figure 2E, and Supplemental Figure 3, C and D) and spleen global myeloid cell populations (Supplemental Figure 3, A and B, and Supplemental Figure 5, A-D) were not quantitatively affected by an absence of graft IL-33, the comparison of $\mathrm{Il33}^{+/+}$and $\mathrm{Il33} 3^{-/-} \mathrm{Bm} 12$ transplanted into $\mathrm{Il33} 3^{+/+}$ B6 recipients by flow cytometry at POD 3 revealed profound early qualitative differences in graft myeloid cells (Figure 3). It is understood that by 3 days after transplant, recipient monocytes infiltrate heart or kidney transplants and transition to monoDCs $\left(\mathrm{CD} 11 \mathrm{~b}^{+}\right.$

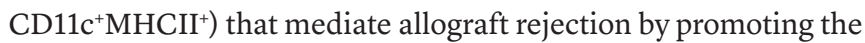
proliferation and survival of $\mathrm{CD}^{+} \mathrm{T}$ cells in the graft (14). Recipient monocytes also differentiate into $\mathrm{CD} 11 \mathrm{~b}^{+} \mathrm{F} 4 / 8 \mathrm{O}^{+} \mathrm{Ly} 6 \mathrm{C}^{\mathrm{hi}}$ and $\mathrm{Ly} 6 \mathrm{C}^{\mathrm{lo}}$ macrophages at early time points after heart transplantation to shape graft pathology and alloimmunity (16). Comparing CD $11 b^{+}$ populations from $\mathrm{Il33} 3^{--} \mathrm{Bm} 12$ to $\mathrm{Il33}{ }^{+/+} \mathrm{Bm} 12$ at POD 3 revealed that in the absence of IL-33, Bm12 grafts had a slight increase in CD11c ${ }^{+}$monoDCs (CD11b $\mathrm{b}^{+} 11 \mathrm{c}^{+} \mathrm{MHCII}^{+}$; Figure 3, A and B). More profound, however, was the increase in proinflammatory Ly6 $\mathrm{C}^{\text {hi }}$ macrophages $\left(\mathrm{CD} 11 \mathrm{~b}^{+} \mathrm{CD} 11 \mathrm{c}^{-} \mathrm{F} 4 / 80^{+}\right.$Ly6C $\mathrm{C}^{\text {hi }}$; Figure $3, \mathrm{C}$ and D), including an $\mathrm{MHCII}^{\mathrm{hi}}$ subset (Figure 3, C and D). Inducible nitric oxide synthase (iNOS) is both a phenotypic marker of proinflammatory macrophages and an effector molecule that enables their functions $(50,51)$. The early increases in inflammatory macrophages persisted in $\mathrm{Il33}^{-/-} \mathrm{Bm} 12$ grafts, as immunofluorescent staining of POD 30 grafts uncovered an increased frequency of $\mathrm{iNOS}^{+} \mathrm{CD} 11 \mathrm{~b}^{+}$cells in those lacking IL-33 (Figure 3, E and F).

Recipient CCR2 $2^{+}$monocyte-derived cells are the mediators of augmented vasculopathy in IL-33-deficient heart grafts. The C-C chemokine receptor type 2 (CCR2) is critical for monocyte tissue entry (52) and subsequent CCR2 ${ }^{+}$monocyte-derived macrophage differentiation into Ly6 $\mathrm{C}^{\text {hi }}$ proinflammatory macrophages that promote tissue damage and contribute to allograft rejection (16, 53). To establish if the increased proinflammatory macrophages observed in the absence of graft IL-33 were propagating accelerated chronic rejection, we assessed $\mathrm{B} 6 \mathrm{WT}$ and $\mathrm{Ccr} 2^{-/-}$recipients of $\mathrm{Il33}^{+/+} \mathrm{BM} 12$ heart transplants at POD 30. IL-33-deficient Bm12 


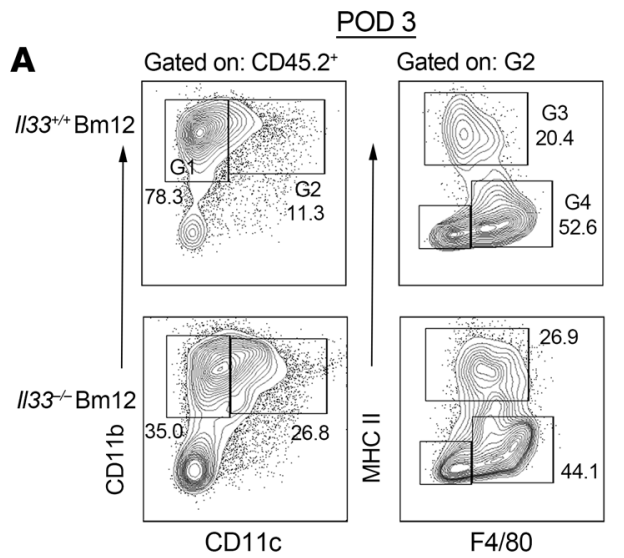

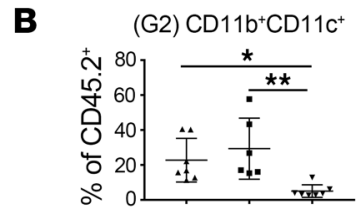

(G3)

$\mathrm{CD} 11 \mathrm{~b}^{+} \mathrm{CD} 11 \mathrm{c}^{+} \mathrm{MHCl}{ }^{\mathrm{hi}}$

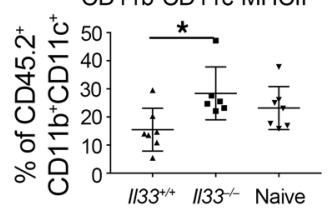

D
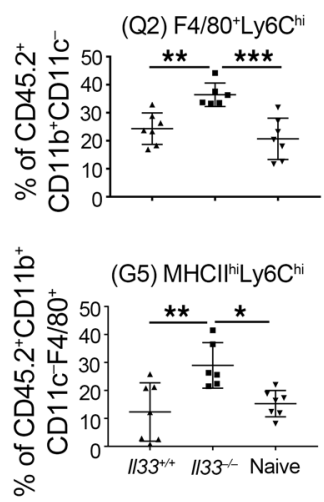

$\mathbf{E}$
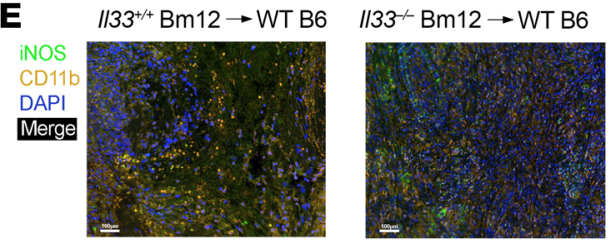

$\mathbf{F}$
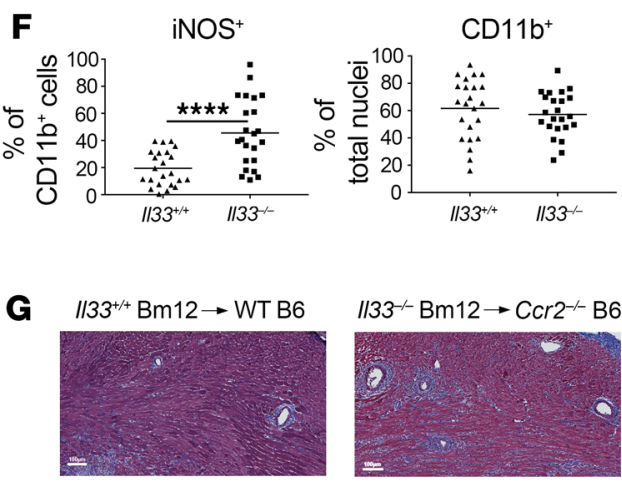

$/ / 33^{-/-} \mathrm{Bm} 12 \rightarrow$ WT B6

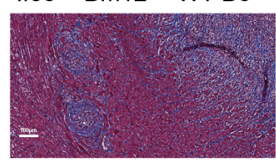

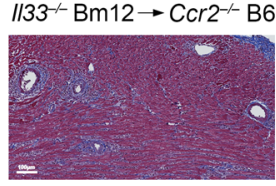

H

Vascular occlusion
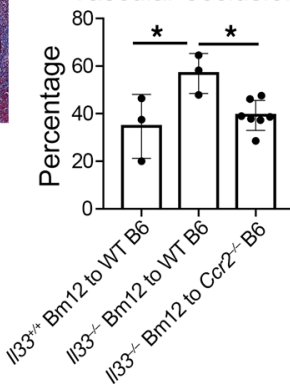

Figure 3. Increased iNOS+ inflammatory myeloid cells cause vasculopathy in IL-33-deficient heart transplants. (A-D) Leukocytes infiltrating $/ / 33^{+/+}$or $1 / 33^{-1-} \mathrm{Bm} 12$ grafts transplanted into WT B6 recipients ( $n=6-7 /$ group) were assessed by flow cytometry on POD 3 . Leukocytes from naive Bm12 hearts were included as baseline controls $(n=4)$. (A) Representative plots of monocyte-derived dendritic cells (monoDCs) in the CD45.2+ gate. (B) Frequency of $\mathrm{CD} 11 \mathrm{~b}^{+} \mathrm{CD} 11 \mathrm{c}^{+}$and $\mathrm{CD} 11 \mathrm{~b}^{+} \mathrm{CD} 11 \mathrm{c}^{+} \mathrm{MHCII}{ }^{\text {hi }}$ cells in the $\mathrm{CD} 45.2^{+}$gate. (C) Representative plots of macrophage subsets in the CD45.2+CD11c $\mathrm{CDD}^{-} \mathrm{b}^{+}$gate. (D)

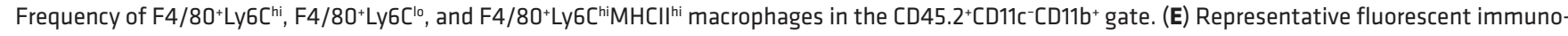
labeling for iNOS (green) and CD11b (orange) and staining with DAPI (blue) in $/ 133^{+/+}$(left panel; $n=5$ ) or $/ / 33^{-I_{-}^{-}}$(right panel; $n=6$ ) Bm12 heart transplants into WT B6 recipients at POD 30. Scale bars: $100 \mu \mathrm{m}$. (F) Quantification of percentage iNOS ${ }^{+}$CD11 $^{+}$and percentage CD11 $\mathrm{b}^{+}$DAPI ${ }^{+}$cells. Graphs depict 3 to 5 values for randomly selected regions from each transplant sample and group mean $\pm \mathrm{SD}$. (G and $\mathbf{H}) / 133^{+/+}$or $/ 133^{-/-}$Bm12 grafts were transplanted into B6 WT or $\mathrm{Cr}^{-1-}$ recipients ( $n=3-7 /$ group). (C) Grafts harvested on POD 30 were evaluated after Masson's trichrome staining for vasculopathy. Scale bars: $100 \mu \mathrm{m}$. (H) Percentage vascular occlusion was quantified, and graphs depict individual values and group mean $\pm \mathrm{SD}$. ${ }^{*} P<0.05$; ${ }^{* *} P<0.01$; ${ }^{* * *} P<0.005$; ${ }^{* * * *} P<0.0001$ by 1-way ANOVA (B, $\mathbf{D}$, and $\left.\mathbf{H}\right)$ or 2-tailed $t$ test with Welch's correction $(\mathbf{F})$.

grafts again exhibited a significant increase in vascular occlusion typical of this time point in WT mice (Figure 2C); however, IL-33deficient allografts in $\mathrm{Ccr}^{-/}$recipients were protected against this accelerated vasculopathy (Figure 3, F and G). In total, our findings to this point establish that graft IL-33 is important to limit early allograft vasculopathy by negatively regulating the generation of proinflammatory macrophages from CCR $2^{+}$recipient cells.

Restoring local IL-33 reduces graft inflammatory myeloid cells and prevents chronic rejection. We have recently revealed that matrixbound nanovesicles (MBV) embedded within the ECM contain bioactive IL-33 (27) and we next assessed if restoring IL-33 locally using MBV could reverse the increase in local inflammatory cells or prevent accelerated chronic rejection. We generated a pliable hydrogel containing IL-33-competent (IL-33+) or IL-33-deficient (IL-33-) MBV and adhered them to grafts immediately after trans- plantation. Hydrogels containing IL-33 ${ }^{+} \mathrm{MBV}$, but not IL-33- $\mathrm{MBV}$, prevented the loss of IL-33-deficient grafts to the accelerated chronic rejection observed before (Figure 4A). Consistent with an antiinflammatory role for local IL-33, we found that IL-33+ $\mathrm{MBV}$ delivery reduced the number of proinflammatory Ly $6 \mathrm{C}^{\text {hi }} \mathrm{F} 4 / 80^{+}$ macrophages, but not $\mathrm{CD} 11 \mathrm{~b}^{+} \mathrm{CD} 11 \mathrm{c}^{+}$monoDCs, in the grafts at POD 3 (Figure 4, B and C, and Supplemental Figure 6). IL-33+ MBV administration at the transplant site did not significantly alter either population in the spleen at this time point (Figure 4, D and E, and Supplemental Figure 6), suggesting that the dominant effect of IL-33 was local. Using hydrogel-based delivery of IL-33 to correct the deficit of graft IL-33 in Il33/- Bm12 grafts, we confirmed that local IL-33 functions to limit the generation of inflammatory macrophages in the allograft early after transplantation. These data also suggest that delivery of regulatory biomolecules, 
A
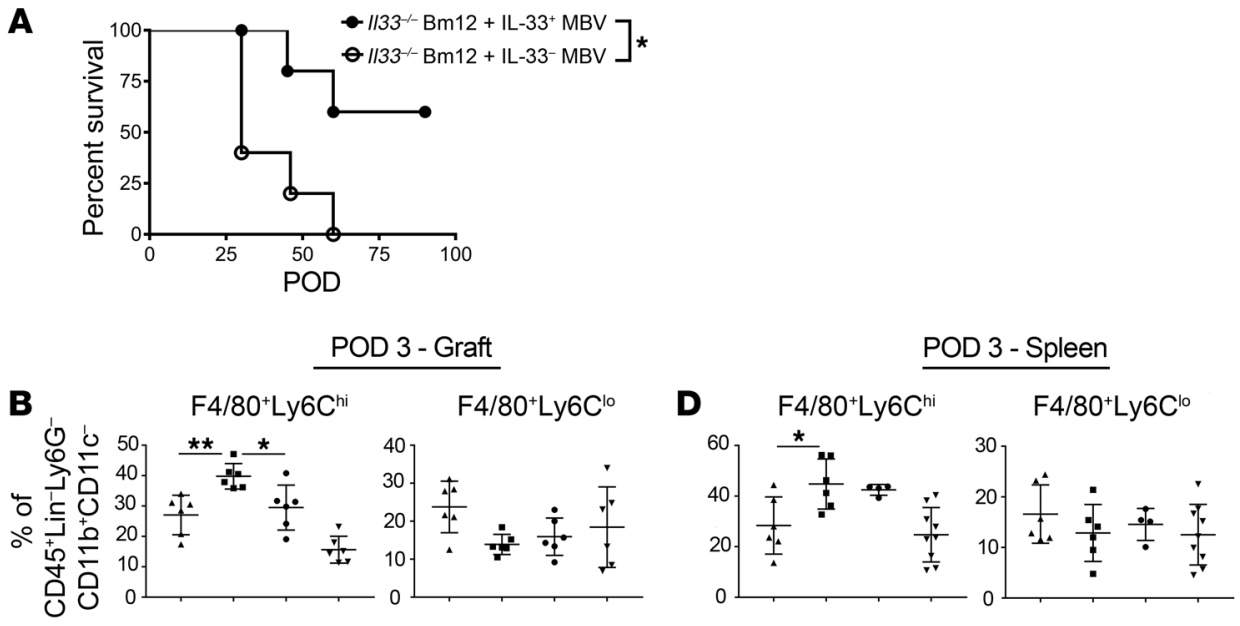

C
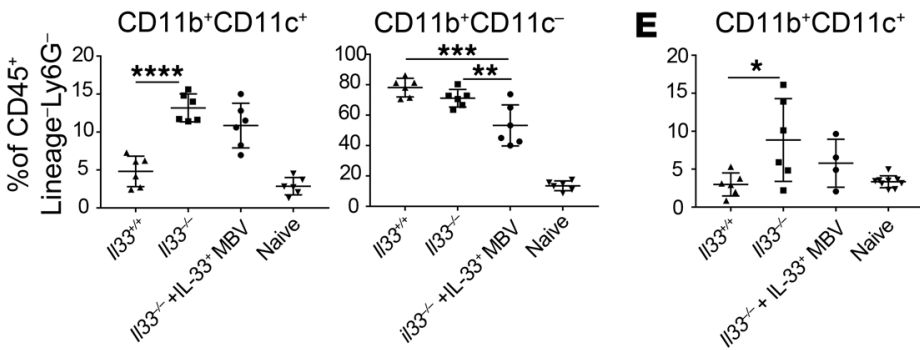

Figure 4. Restoring local IL-33 reduces inflammatory macrophages in the graft and limits chronic rejection of IL-33-deficient allografts. Transplanted $/ 133^{-/-} \mathrm{Bm} 12$ grafts were treated with matrix-bound nanovesicles (MBV) generated from $/ / 33^{-/-} \mathrm{B} 6$ (IL-33- MBV) or $1 / 33^{+/+} \mathrm{B} 6$ (IL-33+ MBV) in hydrogel immediately after transplantation into WT B6 ( $n=6 /$ group). (A) Survival of $1133^{-/-}$Bm12 grafts treated IL-33 $3^{+}$MBV or IL-33- MBV in WT B6 recipients. Graft-infiltrating leukocytes (B and $\mathbf{C}$ ) and splenocytes ( $\mathbf{D}$ and $\mathbf{E})$ at POD 3 from additional groups of WT B6 recipients of $1 / 33^{-/-} \mathrm{Bm} 12$ grafts treated with IL-33+ MBV were compared using flow cytometry to those receiving $1 / 33^{+/+} \mathrm{Bm} 12$ or $1 / 33^{-1-} \mathrm{Bm} 12$ grafts alone. Leukocytes from naive $\mathrm{Bm} 12$ mouse hearts and spleens were also included as controls $(n=6)$. Graphs depict individual values and group mean \pm SD for F4/80+Ly6Chi and F4/80+Ly6 ${ }^{10}$ macrophages in the CD45.2+CD3-CD49b-NK1.1-CD90.2-Ly6C-CD11c-CD11b+ pop-

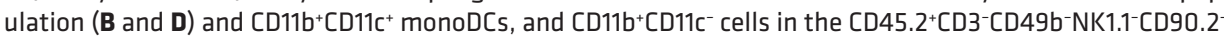
Ly6G- population ( $\mathbf{C}$ and $\mathbf{E}$ ) in the graft and spleen, respectively. ${ }^{*} P<0.05$; $^{* *} P<0.01$; ${ }^{* *} P<0.005$; ${ }^{* * *} P<$ 0.0001 by Kaplan-Meier analysis (A) or 1-way ANOVA (B-E).

such as IL-33, locally to the graft using MBV and hydrogels is feasible and promising for chronic rejection prophylaxis.

IL-33 stimulation poises macrophages through metabolic reprogramming for a regulatory and reparative response. Metabolic remodeling of macrophages is critical to support the energy and metabolite demands required for their response to environmental input during infections and after injury $(54,55)$. Our data above led us to hypothesize that IL-33 may act like IL-4 and IL-10 to augment FA uptake and OXPHOS, which supports the function of regulatory and reparative macrophages $(56,57)$ and tolerogenic DCs (58). To characterize the precise metabolic changes induced by IL-33, we compared the metabolic impact of IL-33 on bone marrow-derived macrophages (BMDMs) to that of the well-characterized BMDM responses to IL-4 or LPS/IFN- $\gamma$ stimulation (50, 51). It was clear that IL-33 behaved similarly to IL- 4 and augmented OXPHOS to increase basal respiration and ATP production (Figure 5, A and B). This effect was in direct contrast to LPS/IFN- $\gamma$ stimulation, which reduced these measures as metabolic activity shifted toward glycolysis (Figure 5, A and B). Global metabolite assessment indicated that IL-33, like IL-4, augmented macro-

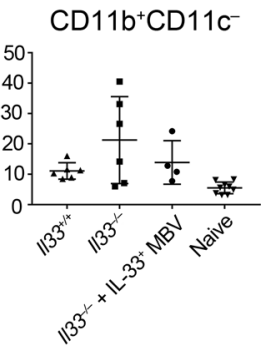

phage ATP generation via OXPHOS using an intact TCA cycle. Both IL-4- and IL-33-stimulated macrophages displayed increased concentrations of carnitine (Supplemental Figure 7A), which is required for FA transport into the mitochondria (55). IL-4 and IL-33 also increased concentrations of $\alpha$-ketoglutarate and glutamate (Supplemental Figure 7B). There was no evidence in IL-33activated BMDMs for increased glucose-6-phosphate (G6P) (Figure $5 \mathrm{C}$ ), the first intermediate of glycolysis, or a buildup of lactate (Figure 5C) that occurs as macrophages use glucose to generate limited ATP via anaerobic glycolysis (55). This was in contrast to LPS/IFN- $\gamma$-activated BMDMs that had high concentrations of NO (Supplemental Figure 7, $\mathrm{D}$ and $\mathrm{E}$ ), G6P, and lactate (Figure 5C). LPS/IFN- $\gamma$-activated BMDMs also displayed the expected $(36,37$, 51) increase in succinate, citrate, and itaconate due to NO-induced disruption of the TCA cycle caused by inhibition of isocitrate dehydrogenase (IDH) (Figure 5C). IL-33-stimulated macrophages were metabolically distinct from IL-4-stimulated macrophages in that they did not display glutamine consumption or accumulate the amino acid ornithine (Supplemental Figure $7 \mathrm{C}$ and ref. 50). Instead, IL-33-stimulated macrophages exhibited high levels of aspartate, malate, fumarate, and increased concentration of arginine relative to both LPS/IFN- $\gamma$ - and IL-4-stimulated macrophages (Supplemental Figure 7A). These metabolites are components of the aspartate-arginosuccinate shunt (AASS), which connects the TCA cycle with the generation of effector metabolites, such as ornithine and citrulline/NO $(50,59,60)$.

CD301 is often used as a marker of IL-4 or alternative macrophage activation and this subset of macrophages is critical for wound repair (61). IL-33 stimulation increased CD301 expression on BMDMs, albeit to a lesser extent relative to IL-4 (Figure 5, D and E). When FA uptake was blocked, a profound inhibition of IL-33-induced CD301 expression resulted (Figure 5, D and E). Although the role for FA uptake and oxidation in macrophage polarization remains controversial (62), our data are consistent with prior studies showing that disruption of FA uptake through inhibitors or loss of the FA translocase CD36 in mice and humans limits the generation and function of immunosuppressive and regulatory myeloid cells $(56,63)$. Thus, IL-33-stimulated macrophages reflect a unique macrophage that is poised through metabolic reprogramming for a regulatory and reparative response 


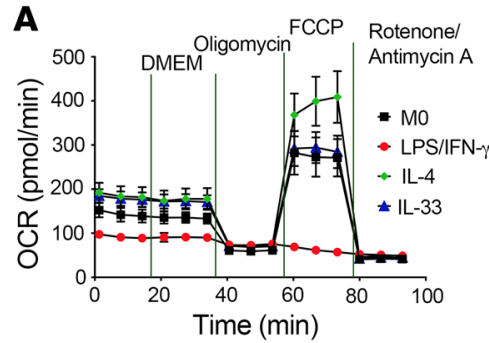

C
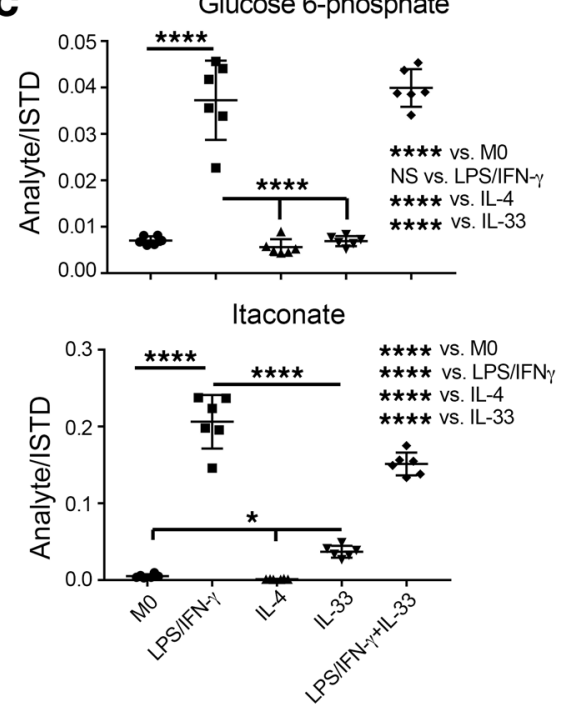

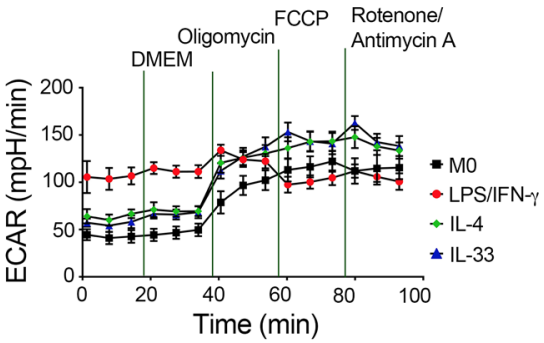

Lactate
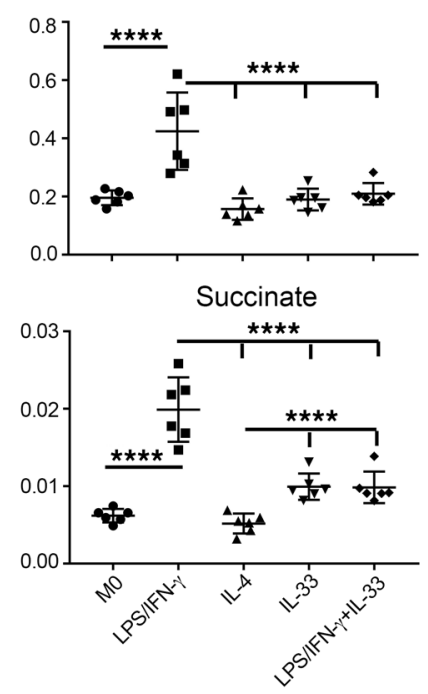
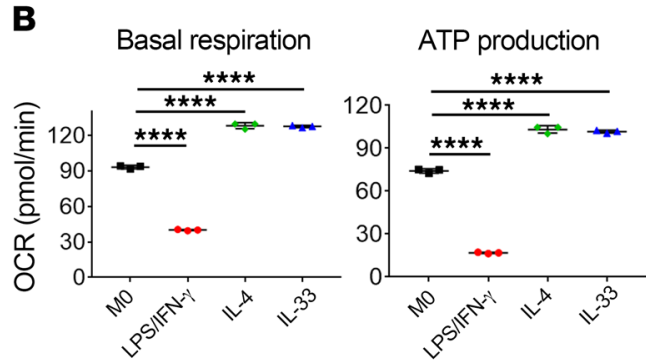

Citrate

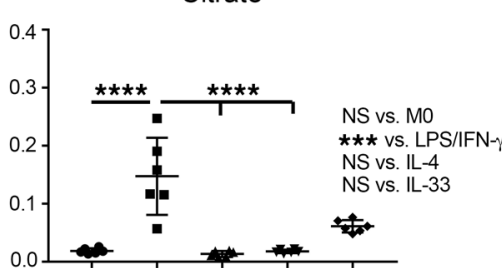

Citrulline

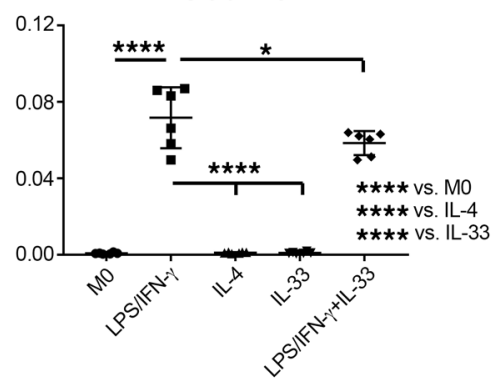

D

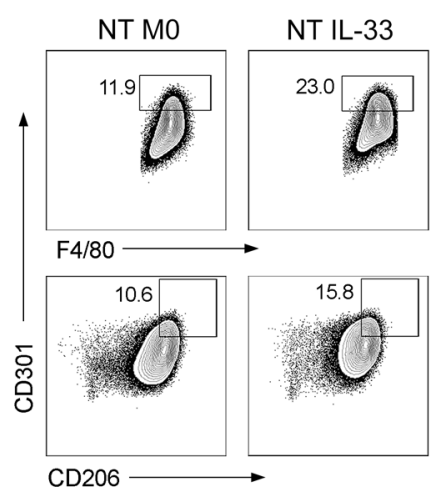

F

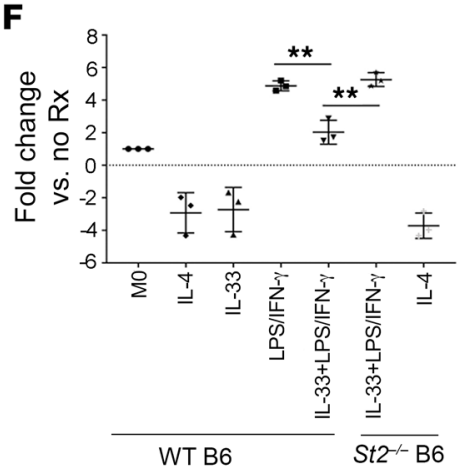

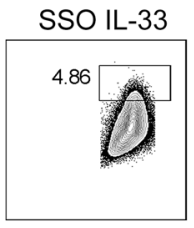

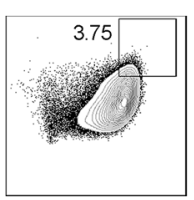

G

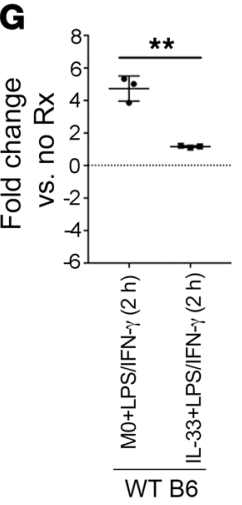

E $\quad$ CD206 ${ }^{+}$CD301

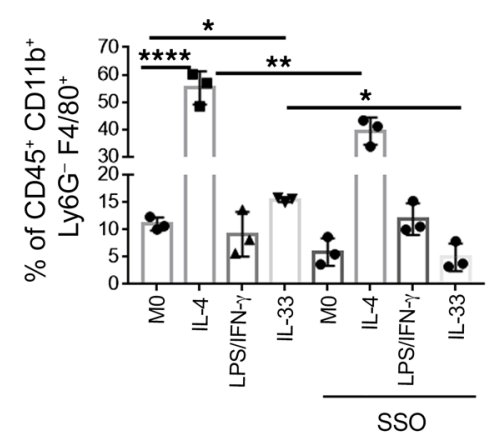

H

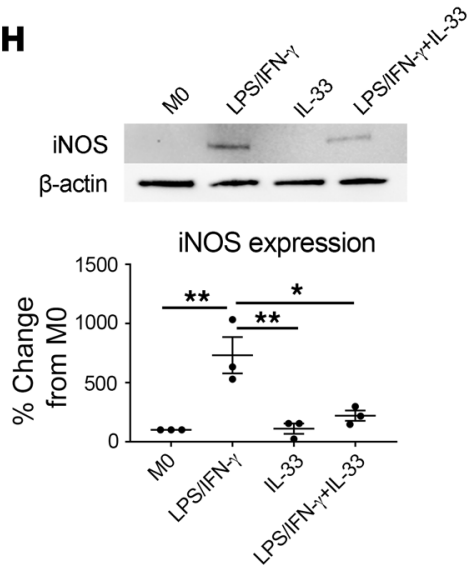


Figure 5. IL-33 mediates an FA-dependent poising of macrophages for differentiation into a reparative and regulatory subset by promoting oxidative phosphorylation and negatively regulating iNOS expression. (A) Oxygen consumption rate (OCR) and extracellular acidification rate (ECAR) were determined for $\mathrm{B} 6$ bone marrow-derived macrophages (BMDMs) left untreated (MO) or stimulated with LPS plus IFN- $\gamma$, or IL-4, or IL-33. One representative experiment of 3 independent experiments is shown. (B) Calculated mitochondrial basal respiration and ATP production for replicates from 1 experiment. (C) Relative concentration (normalized to internal standard [ISTD] and cell number) of TCA metabolites or citrulline in lysates after overnight culture in media alone (MO) or media with LPS plus IFN- $\gamma$, or IL-4, or IL-33; or LPS plus IFN- $\gamma$ and IL-33 determined using liquid chromatography-high-resolution mass spectrometry. $n=6$ samples/ group. ( $\mathbf{D}$ and E) Macrophages stimulated as above alone (non-treated, NT) or with $200 \mu \mathrm{M}$ sulfosuccinimidyl oleate (SSO) for 24 hours were assessed by flow cytometry. (D) Plots of CD45+CD11 + Ly6C $^{-}$F $4 / 80^{+}$-gated cells. (E) Frequency of $\mathrm{CD}^{206} \mathrm{C}^{+} \mathrm{CD} 301^{+}$cells in the $\mathrm{CD} 45^{+} \mathrm{CD} 11 \mathrm{~b}^{+}$Ly6C-F4 $/ 80^{+}$gate. $n=$ 3 samples/group. (F) F4/80+ macrophages from WT or St2 ${ }^{-1-}$ B6 mice were treated as in $\mathbf{C}$ before isolated RNA was assessed for Nos2 by qRT-PCR. (H) WT B6 macrophages were first incubated with IL-33 overnight and then stimulated for 2 hours with LPS plus IFN- $\gamma$ before RNA isolation and Nos 2 qRT-PCR. $n=3$ mice/group. Data are from 1 experiment and representative of 2 completed. (H) Macrophages treated as in $\mathbf{C}$ were assessed by immunoblotting for iNOS. Quantification of immunoblotting mean pixel density was performed using Image) and loading was normalized to $\beta$-actin $(n=3)$. ${ }^{*} P<0.05,{ }^{*} P<0.01,{ }^{* * *} P<0.005,{ }^{* * *} P<0.0001$ by 1 -way ANOVA.

typical of IL-4-activated macrophages, while primed for potential effector responses through an active AASS (see schematic summary in Supplemental Figure 7F).

IL-33 limits proinflammatory signal-induced iNOS expression. Early transplantation-associated IRI and alloimmune-mediated damage of the heart will release IL-33, TLR4 ligands, and proinflammatory cytokines together. Global metabolomic analysis of how IL-33 affected BMDMs receiving simultaneous LPS/IFN- $\gamma$ stimulation revealed that IL-33 did not modulate aerobic glycolysis, as G6P generation was sustained (Figure 5C). However, we observed reduced lactate concentrations, suggesting limited anaerobic glycolysis, as well as decreased levels of citrate and succinate (Figure 5C). Citrulline, which is produced with NO by iNOS from arginine, was also reduced in LPS/IFN- $\gamma$-stimulated BMDMs by the simultaneous presence of IL-33 (Figure $5 \mathrm{C}$ ). NO is critical to macrophage metabolic reprogramming, as it disrupts the electron transport chain by targeting Complex I and IV and decreases IDH $(51,64)$. Decreased IDH activity results in itaconate inhibition of succinate dehydrogenase (SDH), which causes succinate to reach levels that trigger proinflammatory activities (37). These past studies, combined with the observed increased in iNOS ${ }^{+} \mathrm{CD} 11 \mathrm{~b}^{+}$ cells in $\mathrm{Il33}^{-/-} \mathrm{Bm} 12$ grafts (Figure 3, E and F), led us to ask if IL-33 signaling controlled macrophage induction of iNOS. Splenic macrophages receiving simultaneous stimulation with IL-33 and LPS/ IFN- $\gamma$ (Figure 5F) or receiving IL-33 stimulation first, followed by LPS/IFN- $\gamma$ stimulation (Figure $5 \mathrm{G}$ ), had decreased induction of Nos2. BMDMs stimulated with IL-33 and LPS/IFN- $\gamma$ also exhibited a profound reduction in iNOS protein (Figure $5 \mathrm{H}$ ). These data are consistent with the capacity of IL-33 to limit the expression of iNOS in macrophages and prevent metabolic reprogramming supporting proinflammatory functions.

Graft IL-33 targets infiltrating macrophages to stimulate FA uptake and limits macrophage differentiation into an iNOS ${ }^{+}$proin- flammatory subset causing rejection. FA uptake reduces the stimulatory function of tumor-associated DCs and supports the immunosuppressive mechanisms of myeloid-derived suppressor cells $(63,65)$. Likewise, FA uptake contributes to the generation of alternatively activated M2 macrophages that are critical to cardiac tissue repair after ischemia $(56,66)$. IL-33 poised BMDMs for differentiation toward an M2-like state through FA uptake while limiting the induction of iNOS, which is necessary for a metabolic and proinflammatory shift in macrophages toward glycolysis. By comparing $\mathrm{CD}_{11} \mathrm{~b}^{+}$populations from $\mathrm{Il33} 3^{-/-} \mathrm{Bm} 12$ to $\mathrm{Il33} 3^{+/+}$ Bm12 heart transplants at POD 3, we also revealed a significant reduction in levels of FA uptake in the absence of IL-33 in CD11b CD11c ${ }^{-F} 4 / 80^{+}$Ly6C $^{\text {hi }}$ macrophages (Figure 6, A and B). The Ly6C ${ }^{\text {lo }}$ macrophage subset displayed a similar trend toward decreased FA uptake (Figure 6, A and B). These data supported an important role for graft IL-33 in stimulating FA uptake in recipient myeloid cells infiltrating the graft.

Other immune cells, particularly Tregs (29-31, 67-69), express ST2 and could respond to IL-33 and control myeloid cell activation to improve transplant outcomes. We used B6 Lys $M^{\text {Cre }} \times$ $S t 2^{\mathrm{f} / \mathrm{fl}}$ mice, which have targeted deletion of ST2 on $\mathrm{F} 4 / 80^{+}$macrophages, but not Tregs or other immune cells (Supplemental Figure 8), as $\mathrm{Il33}^{+/+} \mathrm{Bm} 12$ heart graft recipients to address this. Using this precise model, we confirmed that IL-33 signaling to macrophages at early time points after transplant limits the differentiation of graft-infiltrating myeloid cells into proinflammatory iNOS ${ }^{+}$ CD20 $6^{\text {lo }}$ macrophages (P1; Figure 6, C-E), but not CD11c ${ }^{+} \mathrm{MHCII}^{\text {hi }}$ monoDCs or CD206 $6^{\text {hi }}$ macrophages (P2; Figure 6, C-E).

The absence of IL-33 signaling in $\mathrm{iNOS}^{+} \mathrm{CD} 206^{\text {lo }}$ macrophages also resulted in augmented levels of iNOS in these cells (P1; Figure 6E) and decreased FA uptake in this macrophage subset (P1; Figure 6F). The deletion of ST2 from recipient macrophages did not, however, alter Tregs in the graft or spleen (Supplemental Figure 9). This loss of IL-33 signaling to macrophages, like the absence of graft IL-33 (Figure 2E), resulted in accelerated rejection of $\mathrm{Il33}^{+/+} \mathrm{Bm} 12$ grafts (Figure $6 \mathrm{G}$ ). In total, these data establish that IL-33 directly targets macrophages to induce a metabolic program supporting FA uptake and regulating iNOS induction that limits the generation of proinflammatory macrophages causing accelerated graft loss.

\section{Discussion}

Myeloid cells are a dominant component of the infiltrating immune cells during acute rejection (70) and increased macrophage numbers present in renal allografts correlates with chronic rejection and poor outcomes (71). Increased graft and circulating myeloid cells are common in samples from recipients with acutely and chronically rejected heart grafts $(72,73)$. Yet only recently have the precise mechanisms by which graft-infiltrating myeloid cells contribute to rejection started to crystalize. Recipient monocytes rapidly infiltrate ischemic allografts and differentiate into monoDC and macrophage subsets that then support the local alloreactive $\mathrm{T}$ cell response $(14,16,17)$. Intracellular alarmins including HMGB1, IL-1 $\alpha$, nuclear DNA, and mitochondrial DNA, as well as ECM components like hyaluronan and heparin sulfate are released upon tissue injury from IRI or alloresponses that then trigger TLRs on myeloid cells to direct their differentiation and 


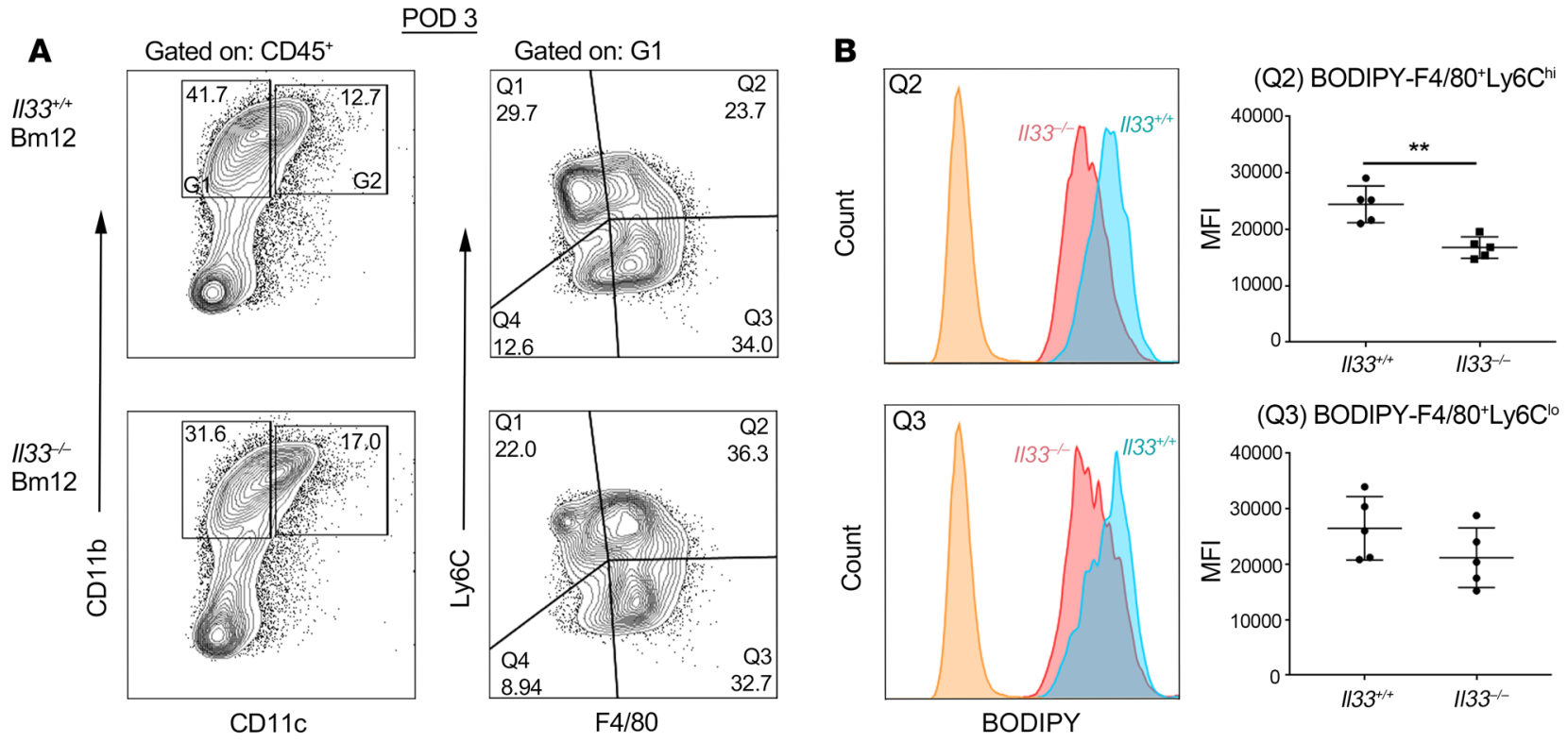

C

POD 3

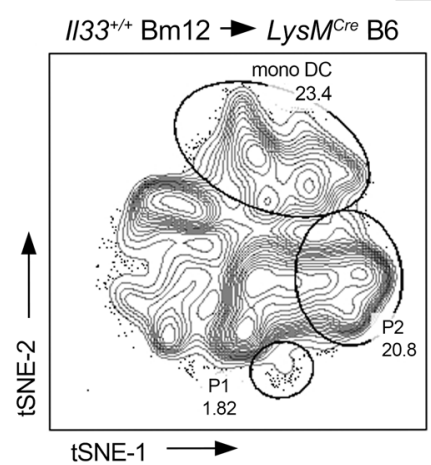

$\| 33^{+/+} \mathrm{Bm} 12 \rightarrow$ LysM $^{\text {Cre }} \times \mathrm{St}{ }^{\mathrm{fl} / f l} \mathrm{~B} 6$

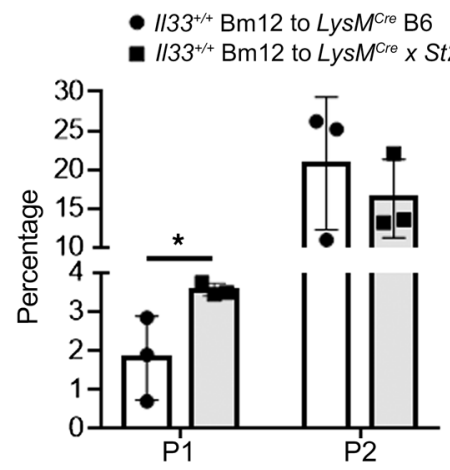

D
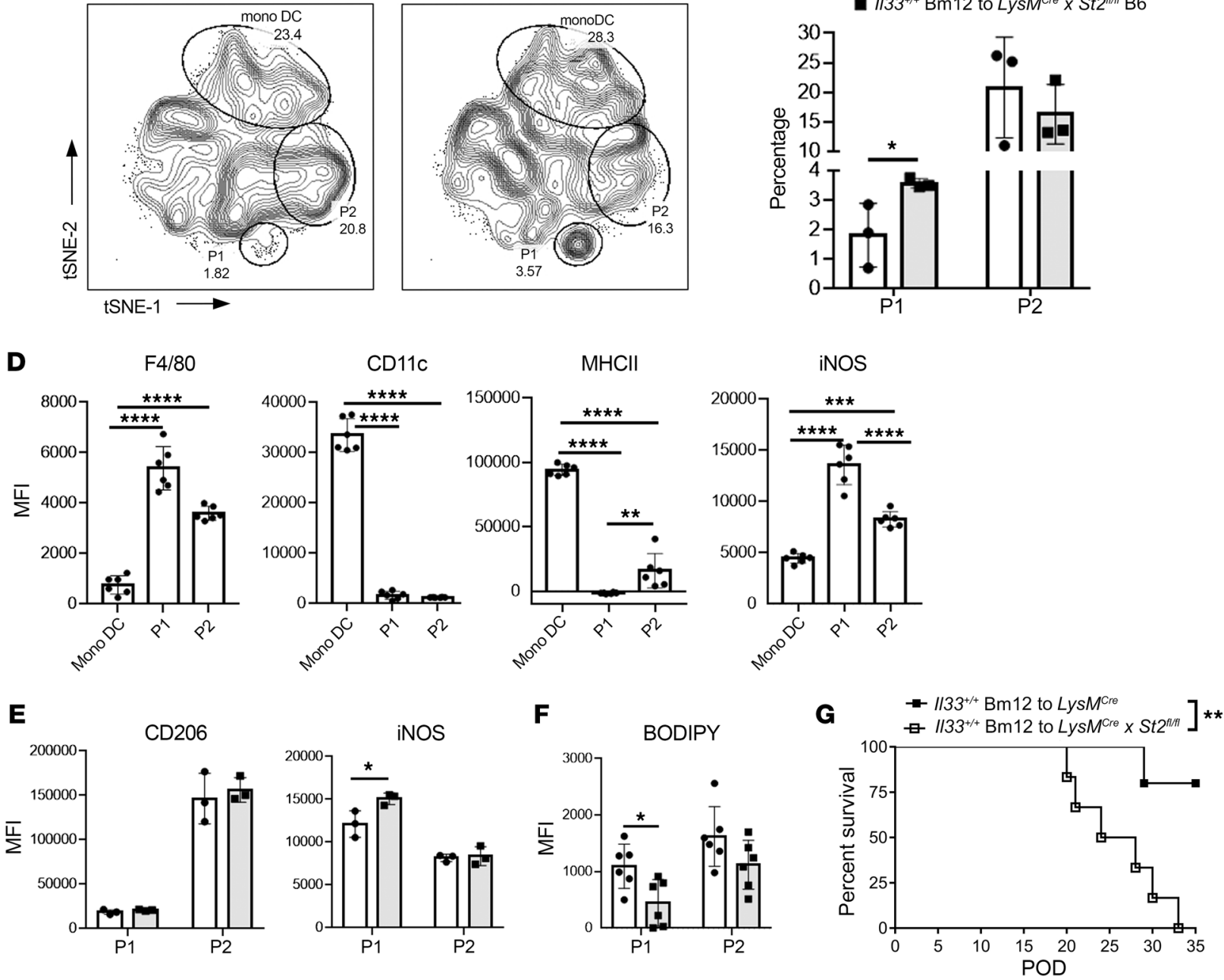
Figure 6. IL-33 targets intragraft myeloid cells to prevent rejection by upregulating their fatty acid uptake and limiting the generation of iNOS+ proinflammatory macrophages. (A) $1 / 33^{+/+}$or $1 / 33^{-/-} \mathrm{Bm} 12$ grafts were transplanted into WT B6 recipients ( $n=5 /$ group). On POD 3 , graft-infiltrating leukocyte fatty acid uptake was assessed using flow cytometry with BODIPY. (B) BODIPY uptake for Q2 (CD45+CD11 b+CD11c-F4/80+Ly6Chi) and Q3 $\left(\mathrm{CD} 45^{+} \mathrm{CD} 11 \mathrm{~b}^{+} \mathrm{CD} 11 \mathrm{C}^{-} \mathrm{F} 4 / 80^{+} \mathrm{Ly} \mathrm{C}^{10}\right)$ populations. Graphs are individual values and group mean $\pm \mathrm{SD}$. (C-E) $/ / 33^{+/+} \mathrm{Bm} 12$ grafts were transplanted into $\mathrm{B} 6$ $L y s M^{\text {cre }}$ or $L y s M^{\text {Cre }} \times S t 2^{f / / f l}$ recipients. On POD 3, graft-infiltrating leukocytes were assessed by flow cytometry. (C) t-Distributed stochastic neighbor embedding (t-SNE) contour plots generated from CD45+CD3-B220-CD11 $\mathrm{b}^{+}$ gated cells ( $n=3$ mice/plot). Population frequency (monoDCs, P1, and P2) for each mouse from $\mathbf{C}$ in t-SNE plot. (D) Mean fluorescence intensity (MFI) for F4/80, CD11c, MHCII, and iNOS for monoDC, proinflammatory (P1), and alternatively activated macrophage (P2) populations. (E) Comparison of MFI for CD206 and iNOS staining between $\mathrm{P} 1$ and $\mathrm{P} 2$ populations from $1 / 33^{+/+} \mathrm{Bm} 12$ grafts transplanted into B6 Lys $M^{\text {cre }}$ (white) or LysM $\mathrm{Mre}^{\mathrm{Cr}} \times \mathrm{St} 2^{\mathrm{fl} / \mathrm{fl}}$ (gray) recipients. Data are from 1 of 2 experiments completed. (F) BODIPY uptake by indicated populations of graft-infiltrating cells in B6 Lys $M^{\text {cre }}$

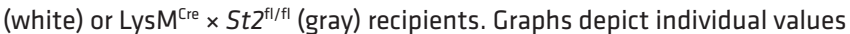
and group mean \pm SD for $\mathrm{CD}^{2} 5^{+} \mathrm{CD}^{-}{ }^{-} \mathrm{B} 22 \mathrm{O}^{-} \mathrm{CD}^{-} 1 \mathrm{~b}^{+} \mathrm{P} 1$ and $\mathrm{P} 2$ gated cells. (C) Survival of $1133^{+/+} B m 12$ grafts transplanted into B6 Lys $M^{\text {Cre }}$ (white) or LysM ${ }^{\text {rre }} \times S t 2^{\mathrm{fl} / \mathrm{fl}}$ recipients ( $n=5$-6/group). ${ }^{*} P<0.05$; ${ }^{* *} P<0.01$; ${ }^{* *} P<$ $0.005 ;{ }^{* * *} P<0.0001$ by 2 -tailed Student's $t$ test (B), 1-way ANOVA (C-E), or Kaplan-Meier analysis (G).

stimulate the type 1 immunity mediating acute and chronic rejection. Recent animal studies have established that graft-infiltrating monocytes recognizing allogenic molecules, such as the polymorphic signal regulatory protein $\alpha(\operatorname{SIRP} \alpha)$, mature into monoDCs that express IL-12 and stimulate $\mathrm{T}$ cell proliferation in the graft (74). Proinflammatory functions of myeloid cells involve reprogramming of their cellular metabolism toward reliance on glycolysis to support their inflammatory functions $(54,75)$. Our data make a convincing case that IL-33 is unique among alarmins and functions as a regulatory alarmin after transplantation to target infiltrating myeloid cells and temper proinflammatory responses by supporting cellular metabolism that enables homeostatic or tissue protective functions $(75,76)$.

Cardiac tissue has limited capacity for repair and cardiomyocytes lost to IRI and alloimmune responses will be replaced by fibrotic scar tissue that prevents cardiac rupture but limits function (77). Thus, methods to limit damage and cardiomyocyte loss after heart transplant are attractive therapeutics. Shortly after cardiac IRI, an early proinflammatory period takes place involving Ly $6 \mathrm{C}^{\text {hi }}$ monocytes and inflammatory macrophages that clear debris by proteolysis and phagocytosis and promote local inflammation through secretion of IL- $1 \beta$, IL- 6 , IL- 12 family members, and TNF- $\alpha$ $(78-80)$. This initial proinflammatory period typically peaks after 3 or 4 days and initiates a period of resolution and remodeling supported by TGF and IL-10 and involves Ly6 $\mathrm{C}^{\mathrm{lo}}$ monocytes and alternatively activated macrophages $(48,80)$. Dysregulation of this process or domination by the proinflammatory period translates into increased pathological remodeling and fibrosis (80). Our characterization of the graft myeloid compartment at 3 days after transplant revealed that IL-33 restricts the proinflammatory capacity of graft-infiltrating, recipient monocyte-derived macrophages that cause chronic rejection-associated vasculopathy and accelerated graft loss. The restoration of local IL-33 limited Ly6 $\mathrm{C}^{\text {hi }}$ macrophages and reduced chronic heart rejection of $\mathrm{Il33^{-/- }} \mathrm{Bm} 12$ grafts. These observations are similar to those when anti-HMGB1 antibodies are delivered after Bm12 heart transplant into B6 recipients. In these studies, targeting HMGB1 reduced the presence of intragraft $\mathrm{CD} 11 \mathrm{~b}^{+} \mathrm{F} 4 / 80^{+}$macrophages, $\mathrm{CD} 11 \mathrm{c}^{+}$monoDCs, and $\mathrm{T}$ cells expressing IFN- $\gamma$ and IL-17A to decrease fibrosis and vessel disease at day 28 after transplantation (20). Based on these considerations, we conclude that a dominant early protective function of IL-33 after heart transplantation is to limit the proinflammatory capacity of local myeloid cells early after heart injury.

Myeloid cells need to be plastic and respond to local stimuli to support host defenses against infections, but also then contribute to the immune regulation and restoration of tissue function. The importance of modulation of macrophage metabolism to carry out these activities has come to light recently (54) and our current studies provide insights into the way that the alarmin IL-33 contributes to regulation of macrophages in the tissues after transplantation. IL-33 stimulation of macrophages augmented OXPHOS and FA uptake, both of which support reparative and regulatory myeloid cell functions. Although IL-33 was not as potent an effector of alternative macrophage activation as IL-4, FA uptake was important for both to increase phenotypic markers of alternative activation.

We have shown previously that IL-33 activates NF- $\mathrm{BB}$ and $\mathrm{p} 38$ in macrophages (27) and monoDCs, which facilitate their ability to expand Th2 cells and Tregs secreting IL-5, IL-10, and IL-13 (30, $69,81)$. These cytokines act on macrophages and other cells in adipose tissue to maintain systemic metabolic homeostasis $(82,83)$. Dahlgren et al. have recently established that Tregs and ST2 ${ }^{+}$conventional type 2 DCs (cDC2s) exist with group 2 innate lymphoid cells (ILC2s) around larger vessels in tissues under homeostatic conditions and $\mathrm{IL}^{-} 33^{+}$stromal cells are crucial to maintain these homeostatic niches (42). Our current observations extend these data and establish that IL-33 may function as part of a homeostasismaintenance or -restoration system in the transplant microenvironment. Specifically, in the absence of IL-33 an uncontrolled early inflammatory response involving increased inflammatory macrophages leads to greatly augmented chronic rejection. Our mechanistic studies showed that IL-33 acts similarly to IL-4 and IL-13 and increases mitochondrial function and FA uptake (56). This is in contrast to TLR4 ligands, like HMGB1 or LPS, which stimulate TLR4 to drive glycolysis and epigenetic modifications enabling inflammatory cytokine production (16). Both ST2 and TLR4 are IL-1R/TLR superfamily members that rely on MyD88 as a signaling adaptor and use NF- $\mathrm{BB}$ and $\mathrm{p} 38$ as downstream effector signaling molecules (26). Both receptors activate mTOR (16). Despite the similarities, however, there are nuanced differences in signaling between TLR4 and ST2 that produce the contrasting functions observed when these closely related pathways are stimulated. One clear difference we identified was the capacity of IL-33 to limit the induction of iNOS in macrophages in vitro and in vivo. Regulation of iNOS is important, as it prevents the NO-induced metabolic reprogramming that enables proinflammatory macrophage functions $(51,64)$. IL-33-exposed myeloid cells can use OXPHOS, where TLR4-stimulated cells will be locked into a metabolic program relying on glycolysis and associated with proinflammatory activities (54). The pharmacological inhibition of glycolysis pre- 
vents DC transition from immature, steady-state DCs to immunogenic DCs (84) and delivery of nanoimmunotherapeutics to macrophages in the allograft and preventing HMGB1-induced glycolysis supports allograft acceptance when delivered with costimulatory blockade (16). Thus, IL-33 represents an endogenous regulatory molecule able to trigger a pathway promoting myeloid cell metabolism toward one supporting injury repair functions and allowing reestablishment of local homeostasis.

Several populations of immune cells that can shape transplant outcomes express ST 2 and respond to IL-33. These include macrophages (27), DCs $(30,42,81), \mathrm{CD}^{+} \mathrm{T}$ cells (85), CD4 ${ }^{+} \mathrm{T}$ cells (86), and Tregs (29-31, 67-69). Previously our work, and that of others, revealed that delivery of rIL-33 promoted cardiac allograft survival by expanding naturally occurring Tregs, including an ST2 ${ }^{+}$ subset, that limited systemic type 1 responses (29). Since then, secretion of the epidermal growth factor amphiregulin (Areg) by IL-33-stimulated ST2 ${ }^{+}$Tregs has been shown to support tissue repair after injury to the skeletal muscle and lung epithelium (31, 67, 68). IL-33 also induces Treg and ILC2 secretion of IL-10 and IL-13 in the adipose tissues and injured lung to limit local inflammation and generate M2 macrophages implicated in tissue repair and homeostasis $(69,82,83)$. ST2 is also expressed by stromal cells and IL-33 has been suggested to be directly protective for cardiac myocytes (87). An important finding from this study was the demonstration that IL-33 from the graft directly targets recipient-derived macrophages to limit their differentiation into a detrimental proinflammatory subset that causes graft loss. These data identify an important protective role for IL-33 and exciting future studies relying on further immune cell- and tissue-specific disruption of ST2 will help us understand how IL-33 coordinates immune and stromal responses to injury after heart transplantation.

Our rodent data suggest that IL-33 levels in the graft increase rapidly but decrease during the posttransplant period. Our examinations of clinical transplant specimens reveal that IL-33 remained increased in grafts exhibiting less vasculopathy and this is suggestive of a beneficial role for sustained local IL-33 that would parallel the protective function revealed for IL-33 in our rodent chronic rejection model. These data are similar to studies by the Mathis group that show that IL-33 expression spikes early after skeletal injury in young mice, but not old mice that have lost IL-33-expressing cells (31). Like our studies in which grafts lacking IL-33 suffer increased chronic rejection, in aging mice poor repair of skeletal muscle was associated with a loss of IL-33expressing cells (31). Cardiac allografts from older donors have increased risk of chronic rejection (88) and it will be important to define if age-related loss of IL-33 may contribute to this risk. We have found that IL-33 is contained in MBV of the ECM of various tissues, including cardiac (27). During the development of cardiac fibrosis the ECM is extensively remodeled (89). Whether this remodeling involves modulation of IL-33 in the ECM to influence the function of local ST2 ${ }^{+}$immune cells will also be a significant question to answer. If $\mathrm{IL}^{-} 33^{+}$cells are indeed reduced in aged donors or if local IL-33 concentrations are depleted during pathological fibrotic remodeling, then our data, showing that local IL-33 delivery reduced the chronic rejection of IL-33-deficient hearts, suggest this type of therapy may be a highly effective chronic-rejection prophylactic.
The revealed biological impacts of IL-33 on macrophages and heart transplantation outcome were profound and our data support the development of targeted IL-33+ therapy to prevent chronic rejection. The concept of stimulating natural reparative pathways is novel relative to other approaches in the DAMP/alarmin space, which instead aim to prevent or block TLR signaling. One advantage of augmenting reparative pathways is that, unlike TLR antagonists or immunosuppressant molecules, this would not stand in the way of normal pathogen responses (18). In addition to heart transplants, other commonly transplanted solid organs, including kidney (90), liver (91), and lung (92), as well as vascular composite allografts (93), all suffer from immunosuppressant-resistant chronic rejection-associated fibrosis and vasculopathy. It will be important to determine if endogenous or delivered IL-33 also acts as a regulatory alarmin in these transplant microenvironments to limit myeloid cell differentiation.

\section{Methods}

Clinical specimens. Pediatric heart transplant-recipient samples were obtained from a prospective study completed at the Children's Hospital of Pittsburgh of UPMC as described previously (94) or an NIAID-funded study, titled "An Observational Cohort Study to Determine the Impact of Alloantibodies and Antibodies to Self-Antigens on Chronic Allograft Function up to 5 years after Pediatric Heart Transplantation." This study enrolled recipients from 9 pediatric heart centers in the United States and Canada. Patients received thymoglobulin induction therapy with subsequent tacrolimus-based immunosuppression plus adjunctive sirolimus or mycophenolate mofetil and underwent serial allograft surveillance EMBs at 1-2 weeks, 2-4 weeks, 2 months, 4 months, 6-7 months, and 10-12 months after heart transplant, and then annually thereafter until the first of either the 5 -year posttransplant study visit, study withdrawal, or end of study follow-up. Patients also underwent EMB if rejection was suspected and to assess resolution following the rejection treatment. Coronary angiograms were obtained at 12 months after transplant and annually thereafter and were analyzed by a single blinded pediatric cardiologist at the study core angiography laboratory at Washington University in St. Louis. Analyzed sera were identified and assessed for IL-33 at times of pathologist-diagnosed acute cellular rejection (ACR) or antibody-mediated rejection (AMR) happening within the first 21-50 days after transplant ( $n=11$ samples from 10 subjects) or suffering mild ( $n=16$ samples from 10 subjects) or severe ( $\mathrm{n}=12$ samples from 5 subjects) chronic rejection-associated coronary artery vasculopathy at least 300 days after transplant. Serum from the Starzl Biorepository for normal healthy nontransplanted adults $(n=8)$ was also assessed. We also separately identified and compared EMB samples from ACR events ( $n=7$ in 4 subjects), AMR events ( $n=7$ in 5 subjects), and no rejection events ( $n=7$ in 6 subjects) in the first year after transplant using immunostaining for IL-33. Multiple EMBs from pediatric heart transplant recipients suffering severe ( $n=8$ in 3 subjects) versus mild CAV ( $n=12$ in 3 subjects) after the first year were also assessed.

Animals. B6, Bm12, Lys $M^{\mathrm{Cre}}$, and Ccr2/- mice were purchased from The Jackson Laboratory. The Il33-/- mice were from S. Nakae (University of Tokyo, Tokyo, Japan) (38). Bm12 Il33/-- mice were generated by backcrossing Bm12 mice 6 times onto the $I l 33^{-/-}$background at the University of Pittsburgh. St2/- mice were originally generated on a BALB/c background (95) and backcrossed 10 times onto the B 6 before 
use. The B6 St $2^{\mathrm{fl}}$ mice were provided by Giorgio Trinchieri (National Cancer Institute, Bethesda, Maryland) and crossed to Lys $M^{\text {Cre }}$ to generate $L y s M^{\mathrm{Cre}} \times S t 2^{\mathrm{t} / \mathrm{fl}}$ mice. All animals were housed in a specific pathogen-free facility maintained by the University of Pittsburgh.

Vascularized heart transplantation. Donor hearts were transplanted into recipients through end-to-side anastomosis of the donor ascending aorta and pulmonary artery to recipient abdominal aorta and inferior vena cava, as described previously (14). In some experiments, MBV isolated from decellularized IL- $33^{+}$mouse intestines, as described previously (96), were diluted in a porcine urinary bladder matrix hydrogel to a final concentration of $1 \mathrm{mg} / \mathrm{mL}$ MBV. Grafts were covered in hydrogel containing $40 \mu \mathrm{g}$ diluted MBV after reperfusion of the graft. The gut was replaced and allowed to resume its normal position around the grafted heart while the MBV in hydrogel stably adhered to the heart surface. Graft function was verified daily by abdominal palpation of heart contractions until the indicated day of harvest.

Isolation of splenic, peritoneal, and graft-infiltrating leukocytes. Mice were anesthetized and perfused with PBS plus 0.5\% heparin via the left ventricle until the fluid exiting the right ventricle did not contain visible blood. Hearts or heart grafts were removed, cut into fragments, and homogenized in a gentleMACS C tube (Miltenyi Biotec) in media containing $350 \mathrm{U} / \mathrm{mL}$ type IV collagenase (Gibco) and $20 \mu \mathrm{g} / \mathrm{mL}$ DNase I (MilliporeSigma) using program $\mathrm{E}$ on the gentleMACS dissociator (Miltenyi Biotec). Single-cell suspensions were then obtained through filtration using a 40- $\mu \mathrm{m}$ cell strainer and centrifuged over a Lympholyte-M (Cedarlane) density gradient. Spleens from recipient or naive mice were isolated and single-cell suspensions generated following mechanical dissociation and RBC lysis. Isolation of splenic macrophages was completed using positive selection with anti-F4/80 MicroBeads (Miltenyi Biotec). Peritoneal cells were obtained by flushing the peritoneal cavity with $1 \mathrm{~mL}$ of cold PBS.

Generation of BMDMs. Murine BMDMs were generated following a procedure similar to that described previously $(50,51,96)$. Briefly, $\mathrm{BM}$ was harvested, washed, and plated in complete medium at $1 \times 10^{6}$ cells $/ \mathrm{mL}$. Cells were allowed to differentiate into macrophages for 7 days in the presence of macrophage colony-stimulating factor (MCSF) (20 ng/mL, BioLegend) or 10\% L929 cell supernatant containing MCSF, with complete media changes every 48 hours. On day 7, macrophages were cultured for $15-18$ hours at $37^{\circ} \mathrm{C}$ and $5 \% \mathrm{CO}_{2}$ in complete media alone (M0) or media supplemented with one or combinations

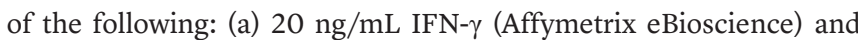
$100 \mathrm{ng} / \mathrm{mL}$ LPS (MilliporeSigma), (b) $20 \mathrm{ng} / \mathrm{mL}$ IL-4 (Invitrogen), and (c) $20 \mathrm{ng} / \mathrm{mL} \mathrm{IL-33} \mathrm{(PeproTech).} \mathrm{After} \mathrm{the} \mathrm{incubation} \mathrm{cells} \mathrm{were}$ washed with sterile PBS and used for experimental assays.

Histological and immunohistochemicalstaining. Naive mouse hearts and heart transplants were formalin-fixed, paraffin-embedded, sectioned $(4 \mu \mathrm{m})$, adhered on glass slides, and stained with H\&E or Masson's trichrome following standard protocols. Using NearCYTE software (http://www.nearcyte.org) as we have described previously (94), blue fibrosis ${ }^{+}$areas $\left(\mathrm{mm}^{2}\right)$ were divided by the whole tissue area $\left(\mathrm{mm}^{2}\right)$ and multiplied by 100 to give a percentage fibrotic area measure. Vascular occlusion was calculated using NearCYTE to quantify the total and open lumen area $\left(\mathrm{mm}^{2}\right)$ for each artery. These values were used to generate a percentage vascular occlusion for each identified artery and a mean percentage vascular occlusion calculated for each recipient. CD3, CD11b, and Foxp3 staining was completed using primary antibodies against CD3 (Abcam, ab1669), CD11b (BD Pharmingen,
550282), Foxp3 (Abcam, ab54501), and then secondary antibodies of biotinylated goat anti-rat (Vector, BA-9400), goat anti-rabbit (Vector, BA-1000), or horse anti-goat (Vector, BA-9500). The total number of $\mathrm{CD}^{+}$, Foxp3 $3^{+}$, or $\mathrm{CD}_{11 \mathrm{~b}^{+}}$cells was calculated manually for each tissue and divided by the total tissue area calculated by NearCYTE to yield a cells/area $\left(\mathrm{mm}^{2}\right)$ measure for each sample.

Quantitative immunofluorescence. Optimal cutting temperature compound-embedded (Thermo Fisher Scientific) frozen mouse heart transplants or naive hearts were sectioned $(6 \mu \mathrm{m})$, placed on glass slides, and stained for CD45 (BD Biosciences, 550539), vimentin (Abcam ab73159), or IL-33 (R\&D Systems, AF3626), and with fluorochrome-conjugated and species-specific secondary antibodies following protocols established in the Center for Biologic Imaging, University of Pittsburgh (http://cbi.pitt.edu) as described previously (29). IL-33 staining of paraffin-embedded EMB sections ( $4 \mu \mathrm{m})$ from pediatric heart transplant recipients was completed following deparaffinization and antigen retrieval similarly to methods we have described previously (94). Intragraft macrophage multiplex immunolabeling of paraffin-embedded transplant sections $(5 \mu \mathrm{m})$ for CD11b (Abcam, ab128797) and iNOS (Thermo Fisher Scientific, PA3030A) was completed following deparaffinization and antigen retrieval using standard protocols and multistep labeling. Full details can be found in the Supplemental Methods.

ELISA. Recipient serum levels of IL-33 were measured using commercial kits (R\&D Systems, DY3625). Samples were isolated at the time of collection, frozen, and stored at $-80^{\circ} \mathrm{C}$ until use. On the day of assessment, samples were batch thawed and determinations of IL-33 completed in triplicate according to manufacturer specifications. The assay limit of detection was $23.44 \mathrm{pg} / \mathrm{mL}$.

Flow cytometry. Isolated splenocytes and graft-infiltrating leukocytes were blocked with heat-inactivated goat serum (5\%), treated with a Live/Dead distinguishing stain, and labeled with combinations of fluorochrome-conjugated antibodies (BD Biosciences, BioLegend, and eBioscience) to distinguish myeloid, T, and B cell populations. Antibodies and their clone numbers against CD45.2 (clone 104), CD19 (1D3), NK1.1 (PK136), (CD45R/B220 (RA3-6B2), CD49b (DX5), CD90.2 (30-H12), Ly6G (1A8); Ly6C (AL-21), CD11b (M1/70), CD11c (HL3), I-A ${ }^{\mathrm{b}}$ (M5/114.15), CD80 (16-10A1), CD86 (24F), SiglecF (E50-2440), CD301 (LOM-14), iNOS (CXNFT), CD206 (MR5D3), Gr-1 (RB6-8C5), F4/80 (BM8), CD45 (30-F11), CD3 (145-2C11), CD4 (GK1.5), CD8 (53-6.7), CD127 (SB/199), CD44 (IM7), CCR7 (4B12), Foxp3 (MF-14), and ST2 (DJ8) were used. BODIPY FL C12 (Invitrogen) was used for FA uptake assessment. Data were acquired with an LSRFortessa (BD Biosciences) or Aurora-10 (Cytek) flow cytometer and analyzed using FlowJo, version 10.1 (BD Biosciences).

Molecular macrophage assessments. Cellular mitochondrial bioenergetics, untargeted metabolomics, electron paramagnetic resonance spectroscopy and spin trapping of NO, qRT-PCR, and Western blot analysis of murine BMDMs were completed using standard techniques described in the Supplemental Methods.

Statistics. Statistical analysis was performed using Prism 7 (Graph$\mathrm{Pad}$ ) and statistical tests used are indicated in the figure legends. $P$ less than 0.05 was considered statistically significant.

Study approvals. Rodent breeding and experimental procedures were approved by and performed in accordance with the guidelines of the Institutional Animal Care and Use Committee of the University of Pittsburgh (protocol 19065158) and complied with the NIH 
Guide for the Care and Use of Laboratory Animals (National Academies Press, 2011). The clinical study protocol was approved by the IRBs of all participating institutions and written informed consent was obtained from parents/guardians with child assent obtained according to local IRB policy.

\section{Author contributions}

ZZ, TL, QL, GSH, JDW, SAW, and HRT conceptualized and designed the research. TL, ZZ, QL, JGB, MV, LRM, GKD, ASR, YCL, HD, JLD, SJM, MWT, GSH, and HRT performed the experiments. ZZ, TL, QL, JGB, MV, LRM, GKD, ASR, SS, MHO, JLD, SJM, SGW, MWT, GSH, SCW, AJD, SFB, and HRT analyzed the data and interpreted the results. ZZ, TL, GKD, MV, LRM, and HRT prepared the figures. TL, ZZ, MV, GKD, GSH, SFB, and HRT drafted and edited the manuscript. TL and ZZ share the first author position, as each contributed key data to the manuscript. The final order was determined based on the work contributed in the laboratory and in manuscript preparation, with TL contributing the most, followed by ZZ.

\section{Acknowledgments}

We thank Anna Lucas for technical assistance and animal husbandry support. We thank Ibraheem Khoury, Benjamin Popp, and Drew Lesniak of the STI Research Histology Services for assistance with EMB assessment. This project used the University of Pittsburgh Genomics Research Core RNA Analysis services and Division of Laboratory Animal Resources. This work was supported by numerous NIH grants: R01AR073527 (to SFB and HRT), R01HL22489 (to HRT), R56AI139327 (to HRT), U01AI104336 (to SAW), R01AI145881 (to MHO), T32CA082084 (to GKD), and F30AI147437 (to GKD). This work benefitted from NIH shared instrumentation grants (S10OD011925 and S10OD023402). ZZ was supported by National Science Foundation of China (grant 81900371) and Natural Science Foundation of Hunan Province (grant 2019JJ50899).

Address correspondence to: Heth R. Turnquist, E1554 Biomedical Science Tower, 200 Lothrop Street, Pittsburgh, Pennsylvania 15261, USA. Phone: 412.624.6695; Email: het5@pitt.edu.
1. Keogh A. Calcineurin inhibitors in heart transplantation. J Heart Lung Transplant.

2004;23(5 suppl):S202-S206.

2. Siu JHY, Surendrakumar V, Richards JA, Pettigrew GJ. T cell allorecognition pathways in solid organ transplantation. Front Immunol. 2018;9:2548.

3. Libby P, Pober JS. Chronic rejection. Immunity. 2001;14(4):387-397.

4. Merola J, Jane-Wit DD, Pober JS. Recent advances in allograft vasculopathy. Curr Opin Organ Transplant. 2017;22(1):1-7.

5. Lodhi SA, Lamb KE, Meier-Kriesche HU. Solid organ allograft survival improvement in the United States: the long-term does not mirror the dramatic short-term success. Am J Transplant. 2011;11(6):1226-1235.

6. Zeng $\mathrm{Q}$, et al. B cells mediate chronic allograft rejection independently of antibody production. J Clin Invest. 2014;124(3):1052-1056.

7. Uretsky BF, et al. Development of coronary artery disease in cardiac transplant patients receiving immunosuppressive therapy with cyclosporine and prednisone. Circulation. 1987;76(4):827-834.

8. Pollack MS, et al. HLA match and other immunological parameters in relation to survival, rejection severity, and accelerated coronary artery disease after heart transplant. Clin Transplant. 1990;4(5):269-275.

9. Zerbe T, et al. Graft atherosclerosis: effects of cellular rejection and human lymphocyte antigen. J Heart Lung Transplant. 1992;11(3 pt 2):S104-S110.

10. Raichlin E, et al. Acute cellular rejection and the subsequent development of allograft vasculopathy after cardiac transplantation. J Heart Lung Transplant. 2009;28(4):320-327.

11. Uehara S, Chase CM, Cornell LD, Madsen JC, Russell PS, Colvin RB. Chronic cardiac transplant arteriopathy in mice: relationship of alloantibody, C4d deposition and neointimal fibrosis. Am J Transplant. 2007;7(1):57-65.

12. Frank R, Molina MR, Goldberg LR, Wald JW, Kamoun M, Lal P. Circulating donor-specific anti-human leukocyte antigen antibodies and complement $\mathrm{C} 4 \mathrm{~d}$ deposition are associated with the development of cardiac allograft vasculopathy. Am J Clin Pathol. 2014;142(6):809-815.

13. Zecher $\mathrm{D}$, et al. Innate immunity alone is not sufficient for chronic rejection but predisposes healed allografts to T cell-mediated pathology. Transpl Immunol. 2012;26(2-3):113-118.

14. Zhuang Q, et al. Graft-infiltrating host dendritic cells play a key role in organ transplant rejection. Nat Commun. 2016;7:12623.

15. Kitchens WH, et al. Macrophage depletion suppresses cardiac allograft vasculopathy in mice. Am J Transplant. 2007;7(12):2675-2682.

16. Braza MS, et al. Inhibiting inflammation with myeloid cell-specific nanobiologics promotes organ transplant acceptance. Immunity. 2018;49(5):819-828.e6.

17. Zhao Y, et al. Macrophage subpopulations and their impact on chronic allograft rejection versus graft acceptance in a mouse heart transplant model. Am J Transplant. 2018;18(3):604-616.

18. Todd JL, Palmer SM. Danger signals in regulating the immune response to solid organ transplantation. J Clin Invest. 2017;127(7):2464-2472.

19. Yang $\mathrm{H}$, et al. A critical cysteine is required for HMGB1 binding to Toll-like receptor 4 and activation of macrophage cytokine release. Proc Natl Acad Sci U S A. 2010;107(26):11942-11947.

20. Zou H, et al. HMGB1 is involved in chronic rejection of cardiac allograft via promoting inflammatory-like mDCs. Am J Transplant. 2014;14(8):1765-1777.

21. Testro AG, et al. Acute allograft rejection in human liver transplant recipients is associated with signaling through Toll-like receptor 4. J Gastroenterol Hepatol. 2011;26(1):155-163.

22. Methe H, Zimmer E, Grimm C, Nabauer M, Koglin J. Evidence for a role of Toll-like receptor 4 in development of chronic allograft rejection after cardiac transplantation. Transplantation. 2004;78(9):1324-1331.

23. Krüger B, et al. Donor Toll-like receptor 4 contrib- utes to ischemia and reperfusion injury following human kidney transplantation. Proc Natl Acad Sci US A. 2009;106(9):3390-3395.

24. Terasaki PI, Cecka JM, Gjertson DW, Takemoto S. High survival rates of kidney transplants from spousal and living unrelated donors. $N$ Engl J Med.1995;333(6):333-336.

25. Matta BM, Reichenbach DK, Blazar BR, Turnquist HR. Alarmins and their receptors as modulators and indicators of alloimmune responses. Am J Transplant. 2017;17(2):320-327.

26. Liew FY, Girard JP, Turnquist HR. Interleukin-33 in health and disease. Nat Rev Immunol. 2016;16(11):676-689.

27. Hussey GS, et al. Matrix bound nanovesicleassociated IL-33 activates a pro-remodeling macrophage phenotype via a non-canonical, ST2-independent pathway. J Immunol Regen Med. 2019;3:26-35.

28. Brunner SM, Schiechl G, Falk W, Schlitt HJ, Geissler EK, Fichtner-Feigl S. Interleukin-33 prolongs allograft survival during chronic cardiac rejection. Transpl Int. 2011;24(10):1027-1039.

29. Turnquist HR, et al. IL-33 expands suppressive

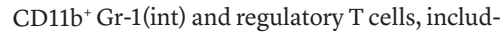
ing ST $2 \mathrm{~L}^{+}$Foxp $3^{+}$cells, and mediates regulatory $\mathrm{T}$ cell-dependent promotion of cardiac allograft survival. J Immunol. 2011;187(9):4598-4610.

30. Matta BM, et al. IL-33 is an unconventional alarmin that stimulates IL-2 secretion by dendritic cells to selectively expand IL-33R/ST2 ${ }^{+}$regulatory T cells. J Immunol. 2014;193(8):4010-4020.

31. Kuswanto W, et al. Poor repair of skeletal muscle in aging mice reflects a defect in local, interleukin-33-dependent accumulation of regulatory $\mathrm{T}$ cells. Immunity. 2016;44(2):355-367.

32. Sanada S, Hakuno D, Higgins LJ, Schreiter ER, McKenzie AN, Lee RT. IL-33 and ST2 comprise a critical biomechanically induced and cardioprotective signaling system. J Clin Invest. 2007;117(6):1538-1549.

33. Seki K, et al. Interleukin-33 prevents apoptosis and improves survival after experimental myo- 
cardial infarction through ST2 signaling. Circ Heart Fail. 2009;2(6):684-691.

34. Abston ED, et al. IL-33 independently induces eosinophilic pericarditis and cardiac dilation: ST2 improves cardiac function. Circ Heart Fail. 2012;5(3):366-375.

35 . Pichery M, et al. Endogenous IL-33 is highly expressed in mouse epithelial barrier tissues, lymphoid organs, brain, embryos, and inflamed tissues: in situ analysis using a novel Il-33LacZ gene trap reporter strain. J Immunol. 2012;188(7):3488-3495.

36. Mills EL, et al. Succinate dehydrogenase supports metabolic repurposing of mitochondria to drive inflammatory macrophages. Cell. 2016;167(2):457-470.e13.

37. Tannahill GM, et al. Succinate is an inflammatory signal that induces IL-1 $\beta$ through HIF-1 $\alpha$. Nature. 2013;496(7444):238-242.

38. Oboki K, et al. IL-33 is a crucial amplifier of innate rather than acquired immunity. Proc Natl Acad Sci U S A. 2010;107(43):18581-18586.

39. McIntyre KR, Seidman JG. Nucleotide sequence of mutant I-A beta bm12 gene is evidence for genetic exchange between mouse immune response genes. Nature. 1984;308(5959):551-553.

40. Schenk S, et al. Alloreactive T cell responses and acute rejection of single class II MHCdisparate heart allografts are under strict regulation by $\mathrm{CD} 4^{+} \mathrm{CD} 25^{+} \mathrm{T}$ cells. JImmunol. 2005;174(6):3741-3748.

41. Yuan X, et al. A novel role of CD4 Th17 cells in mediating cardiac allograft rejection and vasculopathy. J Exp Med.2008;205(13):3133-3144.

42. Dahlgren MW, et al. Adventitial stromal cells define group 2 innate lymphoid cell tissue niches. Immunity. 2019;50(3):707-722.e6.

43. Souders CA, Bowers SL, Baudino TA. Cardiac fibroblast: the renaissance cell. Circ Res. 2009;105(12):1164-1176.

44. Kong P, Christia P, Frangogiannis NG. The pathogenesis of cardiac fibrosis. Cell Mol Life Sci. 2014;71(4):549-574.

45. Yin H, et al. IL-33 prolongs murine cardiac allograft survival through induction of TH2type immune deviation. Transplantation. 2010;89(10):1189-1197.

46. Celli S, Albert ML, Bousso P. Visualizing the innate and adaptive immune responses underlying allograft rejection by two-photon microscopy. Nat Med. 2011;17(6):744-749.

47. Alegre ML, Lakkis FG, Morelli AE. Antigen presentation in transplantation. Trends Immunol. 2016;37(12):831-843.

48. Bajpai G, et al. Tissue resident CCR2- and $\mathrm{CCR}^{+}$cardiac macrophages differentially orchestrate monocyte recruitment and fate specification following myocardial injury. Circ Res. 2019;124(2):263-278.

49. Lavine KJ, et al. Distinct macrophage lineages contribute to disparate patterns of cardiac recovery and remodeling in the neonatal and adult heart. Proc Natl Acad Sci U S A. 2014;111(45):16029-16034.

50. Jha AK, et al. Network integration of parallel metabolic and transcriptional data reveals metabolic modules that regulate macrophage polarization. Immunity. 2015;42(3):419-430.
51. Bailey JD, et al. Nitric oxide modulates metabolic remodeling in inflammatory macrophages through TCA cycle regulation and itaconate accumulation. Cell Rep. 2019;28(1):218-230.e7.

52. Lim JK, Obara CJ, Rivollier A, Pletnev AG, Kelsall BL, Murphy PM. Chemokine receptor Ccr2 is critical for monocyte accumulation and survival in West Nile virus encephalitis. J Immunol. 2011;186(1):471-478.

53. Conde P, et al. DC-SIGN (+) macrophages control the induction of transplantation tolerance. Immunity. 2015;42(6):1143-1158.

54. O'Neill LA, Pearce EJ. Immunometabolism governs dendritic cell and macrophage function. JExp Med. 2016;213(1):15-23.

55. Diskin C, Pålsson-McDermott EM. Metabolic modulation in macrophage effector function. Front Immunol. 2018;9:270.

56. Huang SC, et al. Cell-intrinsic lysosomal lipolysis is essential for alternative activation of macrophages. Nat Immunol. 2014;15(9):846-855.

57. Ip WKE, Hoshi N, Shouval DS, Snapper S, Medzhitov R. Anti-inflammatory effect of IL-10 mediated by metabolic reprogramming of macrophages. Science. 2017;356(6337):513-519.

58. Malinarich F, et al. High mitochondrial respiration and glycolytic capacity represent a metabolic phenotype of human tolerogenic dendritic cells. J Immunol. 2015;194(11):5174-5186.

59. Mills EL, O'Neill LA. Reprogramming mitochondrial metabolism in macrophages as an anti-inflammatory signal. Eur J Immunol. 2016;46(1):13-21.

60. Rodriguez PC, Ochoa AC, Al-Khami AA. Arginine metabolism in myeloid cells shapes innate and adaptive immunity. Front Immunol. 2017;8:93.

61. Shook B, Xiao E, Kumamoto Y, Iwasaki A, Horsley V. CD301b ${ }^{+}$macrophages are essential for effective skin wound healing. J Invest Dermatol. 2016;136(9):1885-1891.

62. Nomura M, et al. Fatty acid oxidation in macrophage polarization. Nat Immunol. 2016;17(3):216-217.

63. Al-Khami AA, et al. Exogenous lipid uptake induces metabolic and functional reprogramming of tumor-associated myeloidderived suppressor cells. Oncoimmunology. 2017;6(10):e1344804.

64. Van den Bossche J, et al. Mitochondrial dysfunction prevents repolarization of inflammatory macrophages. Cell Rep. 2016;17(3):684-696.

65. Herber DL, et al. Lipid accumulation and dendritic cell dysfunction in cancer. Nat Med. 2010;16(8):880-886.

66. Shiraishi M, et al. Alternatively activated macrophages determine repair of the infarcted adult murine heart. JClin Invest. 2016;126(6):2151-2166.

67. Burzyn D, et al. A special population of regulatory $\mathrm{T}$ cells potentiates muscle repair. Cell. 2013;155(6):1282-1295.

68. Arpaia N, et al. A distinct function of regulatory T cells in tissue protection. Cell. 2015;162(5):1078-1089.

69. Liu Q, et al. IL-33-mediated IL-13 secretion by ST2 ${ }^{+}$Tregs controls inflammation after lung injury. JCI Insight. 2019;4(6):123919.

70. Hancock WW, Thomson NM, Atkins RC. Com- position of interstitial cellular infiltrate identified by monoclonal antibodies in renal biopsies of rejecting human renal allografts. Transplantation. 1983;35(5):458-463.

71. Toki $\mathrm{D}$, et al. The role of macrophages in the development of human renal allograft fibrosis in the first year after transplantation. Am J Transplant. 2014;14(9):2126-2136.

72. Zhao DX, Hu Y, Miller GG, Luster AD, Mitchell RN, Libby P. Differential expression of the IFN-gamma-inducible CXCR3-binding chemokines, IFN-inducible protein 10, monokine induced by IFN, and IFN-inducible T cell alpha chemoattractant in human cardiac allografts: association with cardiac allograft vasculopathy and acute rejection. JImmunol. 2002;169(3):1556-1560.

73. Salama M, Andrukhova O, Roedler S, Zuckermann A, Laufer G, Aharinejad S. Association of $\mathrm{CD}_{14}{ }^{+}$monocyte-derived progenitor cells with cardiac allograft vasculopathy. J Thorac Cardiovasc Surg. 2011;142(5):1246-1253.

74. Oberbarnscheidt MH, et al. Non-self recognition by monocytes initiates allograft rejection. J Clin Invest. 2014;124(8):3579-3589.

75. Eming SA, Wynn TA, Martin P. Inflammation and metabolism in tissue repair and regeneration. Science. 2017;356(6342):1026-1030.

76. Caputa G, Castoldi A, Pearce EJ. Metabolic adaptations of tissue-resident immune cells. Nat Immunol. 2019;20(7):793-801.

77. Yellon DM, Hausenloy DJ. Myocardial reperfusion injury. N Engl J Med. 2007;357(11):1121-1135.

78. Frangogiannis NG. Inflammation in cardiac injury, repair and regeneration. Curr Opin Cardiol. 2015;30(3):240-245.

79. Dutta P, Nahrendorf M. Monocytes in myocardial infarction. Arterioscler Thromb Vasc Biol. 2015;35(5):1066-1070.

80. Wynn TA, Vannella KM. Macrophages in tissue repair, regeneration, and fibrosis. Immunity. 2016;44(3):450-462.

81. Turnquist HR, et al. mTOR and GSK-3 shape the $\mathrm{CD}_{4}{ }^{+} \mathrm{T}$-cell stimulatory and differentiation capacity of myeloid DCs after exposure to LPS. Blood. 2010;115(23):4758-4769.

82. Miller AM, et al. Interleukin-33 induces protective effects in adipose tissue inflammation during obesity in mice. Circ Res. 2010;107(5):650-658.

83. Molofsky AB, et al. Innate lymphoid type 2 cells sustain visceral adipose tissue eosinophils and alternatively activated macrophages. J Exp Med. 2013;210(3):535-549.

84. Jantsch J, et al. Hypoxia and hypoxia-inducible factor-1 alpha modulate lipopolysaccharideinduced dendritic cell activation and function. JImmunol. 2008;180(7):4697-4705.

85. Bonilla WV, et al. The alarmin interleukin-33 drives protective antiviral $\mathrm{CD} 8^{+} \mathrm{T}$ cell responses. Science. 2012;335(6071):984-989.

86. Baumann C, et al. T-bet- and STAT4-dependent IL-33 receptor expression directly promotes antiviral Th1 cell responses. Proc Natl Acad Sci U S A 2015;112(13):4056-4061.

87. Rui T, Tang Q. IL-33 attenuates anoxia/reoxygenation-induced cardiomyocyte apoptosis by inhibition of PKC $\beta / J N K$ pathway. PLoS One. 2013;8(2):e56089. 
88. Axtell AL, et al. The effect of donor age on posttransplant mortality in a cohort of adult cardiac transplant recipients aged 18-45. Am J Transplant. 2019;19(3):876-883.

89. Fan D, Takawale A, Lee J, Kassiri Z. Cardiac fibroblasts, fibrosis and extracellular matrix remodeling in heart disease. Fibrogenesis Tissue Repair. 2012;5(1):15.

90. Hart A, et al. OPTN/SRTR 2015 annual data report: Kidney. Am J Transplant. 2017;17(suppl 1):21-116.
91. Duffy JP, et al. Long-term patient outcome and quality of life after liver transplantation: analysis of 20-year survivors. Ann Surg. 2010;252(4):652-661.

92. Verleden SE, et al. Restrictive chronic lung allograft dysfunction: Where are we now? J Heart Lung Transplant. 2015;34(5):625-630.

93. Krezdorn N, et al. Chronic rejection of human face allografts. Am J Transplant. 2019;19(4):1168-1177.

94. Mathews LR, et al. Elevated ST2 distinguishes incidences of pediatric heart and small bowel transplant rejection. Am J Transplant. 2016;16(3):938-950.

95. Townsend MJ, Fallon PG, Matthews DJ, Jolin HE, McKenzie AN. T1/ST2-deficient mice demonstrate the importance of T1/ST2 in developing primary T helper cell type 2 responses. J Exp Med. 2000;191(6):1069-1076.

96. Huleihel L, et al. Matrix-bound nanovesicles within ECM bioscaffolds. Sci Adv . 2016;2(6):e1600502. 